\title{
CrystEngComm
}

Cite this: CrystEngComm, 2014, 16 4069

Received 14th February 2014 Accepted 5th March 2014

DOI: $10.1039 / c 4 c e 00321 \mathrm{~g}$

www.rsc.org/crystengcomm

\section{Rational design of metal-organic frameworks with anticipated porosities and functionalities $\dagger$}

\author{
Muwei Zhang, ${ }^{a}$ Mathieu Bosch, ${ }^{a}$ Thomas Gentle III ${ }^{a}$ and Hong-Cai Zhou*ab
}

\begin{abstract}
Metal-organic frameworks have emerged as a new category of porous materials that have intriguing structures and diverse applications. Even though the early discovery of new MOFs appears to be serendipitous, much effort has been made to reveal their structure-property relationships for the purpose of rationally designing novel frameworks with expected properties. Until now, much progress has been made to rationalize the design and synthesis of MOFs. This highlight review will outline the recent advances on this topic from both our and other groups and provide a systematic overview of different methods for the rational design of MOFs with desired porosities and functionalities. In this review, we will categorize the recent efforts for rational MOF design into two different approaches: a structural approach and a functional approach.
\end{abstract}

\section{Introduction}

Metal-organic frameworks (MOFs), also known as porous coordination polymers (PCPs), are an important category of crystalline materials that exist as well-defined supramolecular architectures, in which the metal-containing units are coordinatively connected by ditopic or polytopic organic

${ }^{a}$ Department of Chemistry, Texas A\&M University, College Station, Texas, 77842, USA. E-mail: zhou@mail.chem.tamu.edu; Fax: +1 979845 1595; Tel: +1 9798454034 ${ }^{b}$ Department of Materials Science and Engineering, Texas A\&M University, College Station, Texas, 77842, USA

$\dagger$ Electronic supplementary information (ESI) available: The chemical structures of all of the ligands, abbreviations and acronyms, and the highresolution figures of some selected MOF structures can be found in the ESI. See DOI: $10.1039 / \mathrm{c} 4 \mathrm{ce} 00321 \mathrm{~g}$

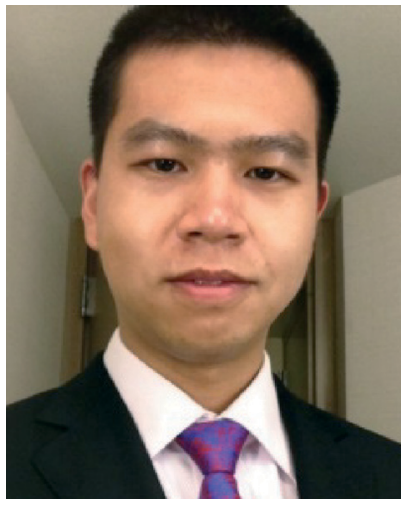

Muwei Zhang
Muwei Zhang was born in the city of Xiantao, Hubei, China in 1988. After he received his B.S. degree in Chemistry from Wuhan University in 2009, he continued his Ph.D. study at Texas A\&M University under the guidance of Prof. Hong-Cai "Joe" Zhou. He is currently a fifth-year Ph.D. candidate. His research is focused on the rational design of metal-organic frameworks (MOFs) and porous polymer networks (PPNs) for clean energy applications. He is particularly interested in the symmetryguided synthesis of porous materials and reticular chemistry. linkers to exhibit a variety of infinite three dimensional networks with potential inner porosity. ${ }^{1-3}$ Due to their fascinating structures and diverse applications, they have attracted a great amount of attention in the past few decades. MOFs are promising materials for many different applications, such as gas storage $\mathrm{e}^{4-6}$ and separation, ${ }^{7-9}$ environmental conservation, ${ }^{10-12}$ heterogeneous catalysis, ${ }^{13,14}$ luminescent materials $^{15,16}$ and biomedical materials. ${ }^{17,18}$ Since the emergence of several representative MOF compounds such as MOF- $5^{19}$ and HKUST-1, ${ }^{20}$ more than 20000 MOF structures have been reported and studied to date. ${ }^{21}$ Due to the extraordinarily large extent of variability of both metal-containing units and organic linkers, it appears that an inexhaustible amount of MOFs could theoretically be designed and synthesized. This, on the other hand, makes it more difficult for researchers to

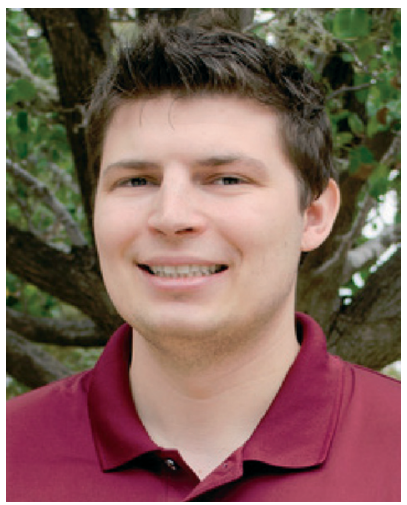

Mathieu Bosch
Mathieu Bosch was born in Hampton, Virginia. He worked full time for several years before attending the University of Houston starting in 2010. He graduated Summa Cum Laude, with honors in Chemistry in May 2013 and has been a graduate student at Texas A\&M University since then, studying under Prof. Hong-Cai "Joe" Zhou. His research interests lie in the synthesis and crystallographic characterization of novel advanced porous materials for catalysis and energy storage. 
search for a rational way to design a framework with properties exactly suitable for a specific use. In fact, since the emergence of MOFs, targeted syntheses of MOFs with desired framework cavities and customized framework functionalities have been a challenging problem..$^{22-26}$ It is highly desirable to rationally design a novel MOF whose properties can be predicted from its basic constructional units before the framework is synthesized.

The purpose of rationally designing MOFs is to achieve accurate control of the framework porosity and functionality from the molecular level for a customized interaction between the framework and its guest molecules. Though it is challenging, chemists have been searching for solutions to overcome these difficulties. Thanks to the tunable nature and convenient functionalization processes of MOFs, chemists are close to achieving this goal. Reticular synthesis, ${ }^{27,28}$ introduced by Yaghi and O'Keeffe's groups, is a method in which, based on an existing MOF structure, the combination of an elongated organic linkers with the same metal-containing secondary building units (SBUs) will possibly result in an isoreticular framework with the same topology but larger pore size. Designing ligands ${ }^{29,30}$ with various geometries and functional groups has enabled us to tentatively control the topology and functionality of the resulting framework. Post-synthetic modifications (PSMs) ${ }^{31,32}$ of MOFs, on the other hand, have assisted us in introducing desired functional groups into the framework in a convenient way. Symmetry-guided design, ${ }^{25}$ a more recent work from our group, suggested that the scrutiny of simple mineral structures can lead to the design and synthesis of significantly porous frameworks with desired topology and cavity size. In consideration of the rapidly growing research on this topic in the past few years, herein we would like to write this review to highlight the recent advances on rational MOF design from our and other research groups and provide a general outline of different practical ways to construct MOFs with expected properties.
It is widely accepted that MOFs are renowned for both their variety of captivating structures and their widespread potential applications. ${ }^{1,21}$ Herein, we would like to categorize recent advances on rational MOF design into two different approaches: a structural approach and a functional approach. The structural approach includes the rational design of MOFs with expected structures, topologies and porosities, while the functional approach involves the rational design of MOFs with desired functionalities for a particular application (Scheme 1). All of these approaches will be elaborated upon in the following sections.

\section{Rational design of MOFs with anticipated structures and porosities}

\subsection{Symmetry-guided design of highly porous MOFs}

Even though the composition of MOFs appears to be simple, that is, the organic linkers and metal-containing clusters (or SBUs), it is still rather difficult to accurately predict the structure, porosity, and topology of the resulting framework with a given organic linker and a metal source. First, the organic ligands can adopt many different conformations, which, upon incorporation into the framework, will result in polymorphic MOFs with completely different structures and properties. ${ }^{33,34}$ Second, the metal ions can form different metal clusters, which will result in different MOF structures as well. $^{35,36}$ Third, when an elongated ligand was utilized, the resulting MOF will, in many cases, suffer from undesired and unpredictable framework interpenetration. ${ }^{27,37}$ It is still quite problematic to control the framework interpenetration simply by tuning the solvothermal reaction conditions. ${ }^{38-40}$ These limitations made it rather challenging to design MOFs with cavities that possess the desired size, shape, and function.

The introduction of framework topology has brought novel insights into the rational design of highly porous MOFs. ${ }^{25}$ Based on a given topology, it is easy for one to determine

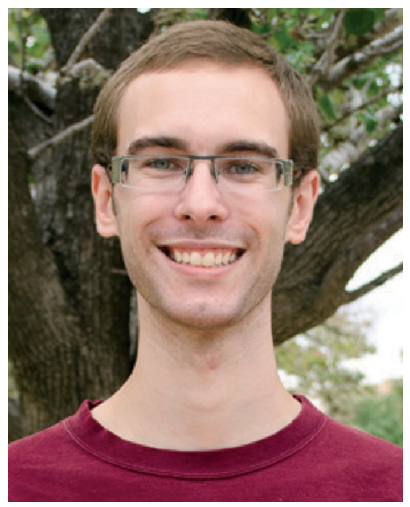

Thomas Gentle III
Tommy Gentle was born in Midland, Michigan in 1992. He earned his B.S. degree in Chemistry from the University of Saint Thomas in Saint Paul, Minnesota in 2013 where his focus was on organic synthesis. In 2013, he joined Zhou's group at Texas A\&M University to obtain his Ph.D. degree in Chemistry. His research interests lie in ligand design for metal-organic frameworks.

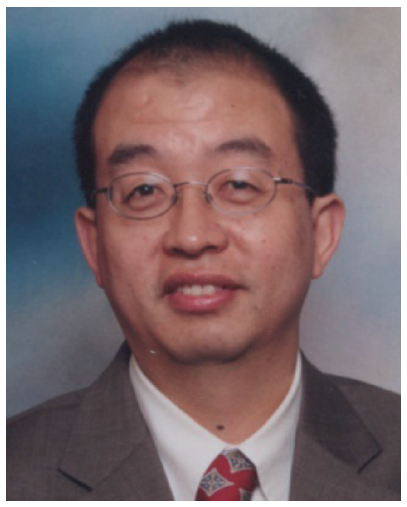

Hong-Cai Zhou
Hong-Cai "Joe" Zhou obtained his B.S. degree from Beijing Normal University in 1984 and his Ph.D. degree in 2000 from Texas A\&M University under the supervision of $F$. A. Cotton. After a postdoctoral stint at Harvard University with $R$. $H$. Holm, he joined the faculty of Miami University, oxford, in 2002. He rose to the rank of full professor within six years and moved to Texas A\&M University in 2008. He was a guest editor (co-editors: Jeffrey Long and Omar Yaghi) for the first Chemical Reviews thematic issue on Metal-Organic Frameworks in 2012. He has been an associate editor for the ACS journal Inorganic Chemistry since June 2013. 


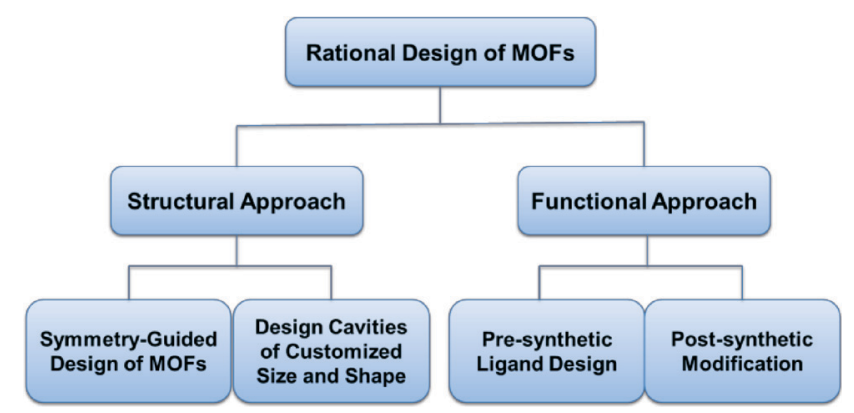

Scheme 1 Recent efforts for the rational design of MOFs can be categorized into two different catalogs: a structural approach and a functional approach.

many important properties of a framework, such as the connectivity of its nodes, the shape and potential size of its cavities, and the possibility of this framework to endure selfinterpenetration. ${ }^{41-44}$ Previous efforts on MOF topochemistry typically relied on the analysis of framework topology after the MOF structure was resolved. However, the symmetryguided design implies it is possible to achieve a bottom-up design with the desired network connectivity and topology prior to the construction of MOFs. ${ }^{25}$

2.1.1 Symmetry-guided synthesis of MOFs with desired topology. The fluorite topology ${ }^{45}$ (flu) is especially interesting for MOF construction for several reasons. First, the fluorite topology is the default topology that connects the tetrahedral nodes and cubic nodes by only one type of link. This will eliminate possibilities of forming MOFs with other topologies under the same conditions, which makes it possible to predict the structure of the resulting MOF before it is made. ${ }^{46,47}$ Second, the flu net is unlikely to undergo selfinterpenetration. This is extremely helpful for the construction of MOFs with large cavities, since the elongation of organic linkers will typically result in framework interpenetration. ${ }^{48}$ Third, MOFs with this topology may exhibit high porosity. A close examination of the fluorite reveals its structure as a cubic close packing (ccp) of calcium where all its tetrahedral interstitial holes are filled by fluoride (Fig. 1(a)). It should be noted that all of the octahedral interstitial holes remain unoccupied (Fig. 1(b)). By augmenting the fluoride nodes with a rigid tetrahedral ligand (Fig. 1(c)) and substituting the calcium nodes by an 8-connected metal-containing cluster, a MOF with the same topology but considerably enlarged interstitial octahedral cavities will be generated (Fig. 1(d)). ${ }^{25}$

In order to construct a framework with fluorite topology, the key step is to find the two components that are symmetrically complementary with each other: a 4-connected ligand that can be topologically represented as a tetrahedral node, ${ }^{49}$ and an 8-connected metal-containing SBU as a cubic node. In this work, zirconium polyoxo clusters have attracted our attention for their abundance, ${ }^{50}$ stability, ${ }^{51}$ and tunable connectivity. ${ }^{36}$ PCN-521 (PCN represents porous coordination network) consists of 8-connected $\left[\mathrm{Zr}_{6}\left(\mu_{3}-\mathrm{OH}\right)_{8}(\mathrm{OH})_{8}\right]$ clusters combined with the MTBC ligand $\left(\right.$ MTBC $=4^{\prime}, 4^{\prime \prime}, 4^{\prime \prime \prime}, 4^{\prime \prime \prime \prime}-$ methanetetrayltetrabiphenyl-4-carboxylate, see Section 1 in (a)

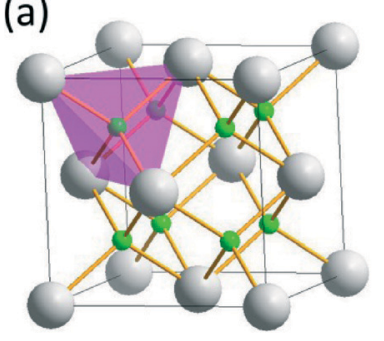

(c)

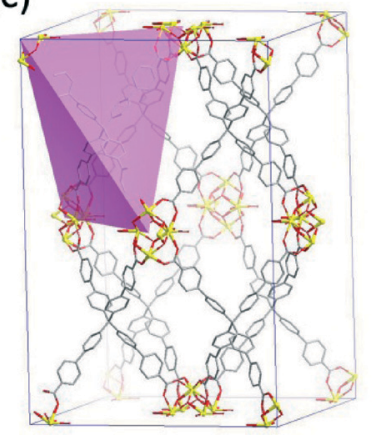

(b)

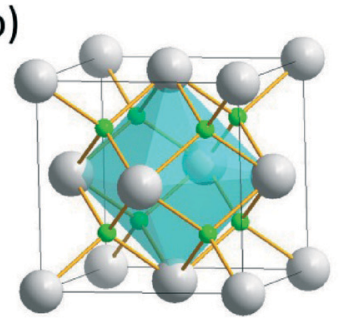

(d)

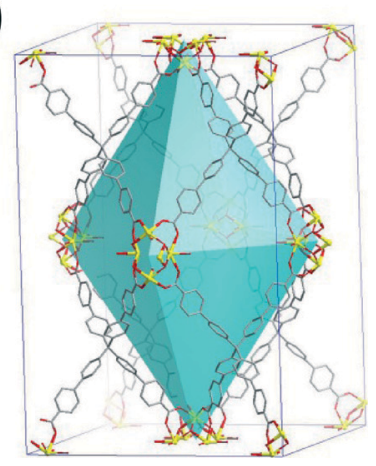

Fig. 1 (a, b) The graphic representation of the fluorite structure. The pink and turquoise polyhedra represent the tetrahedral and octahedral interstitial cavities from the ccp of $\mathrm{Ca}^{2+}$, respectively. Color scheme: $\mathrm{Ca}$, gray; and $\mathrm{F}$, green. (c, d) The structure of PCN-521, a fluoritetopology MOF. C, gray; O, red; and $\mathrm{Zr}$, yellow. The size of the cavity in $\mathrm{PCN}-521$ is $20.5 \times 20.5 \times 37.4 \AA$. (Reprinted from ref. 25 with permission, copyright 2014 John Wiley \& Sons.)

the ESI $\dagger$ ). This MOF possesses an expected flu topology denoted as $\left(4^{12} \cdot 6^{12} \cdot 8^{4}\right)\left(4^{6}\right)_{2}$. After augmentation of the 4-connected tetrahedral nodes by the MTBC ligand, the "octahedral interstitial cavity" of this framework was significantly amplified to the size of $20.5 \times 20.5 \times 37.4 \AA$ (Fig. 1(d)), providing the largest cavity of any MOFs made from tetrahedral linkers. ${ }^{25}$ Due to the chemical resemblance between zirconium and hafnium, an isostructural framework, PCN-523, with hafnium-containing SBUs, has also been reported. It should be noted that PCN-521 also possesses the largest solvent accessible volume $(78.50 \%)$ and Brunauer-EmmettTeller (BET) surface area $\left(3411 \mathrm{~m}^{2} \mathrm{~g}^{-1}\right)$ among all of the MOFs with tetrahedral ligands. It is the first example of a noninterpenetrated MOF constructed from the MTBC ligand. ${ }^{52-54}$

Another 8-connected SBU that is appropriate for the construction of the fluorite-topology MOF is the $\left.\left[\mathrm{M}_{4}\left(\mu_{4}-\mathrm{Cl}\right) \text { (tetrazolate }\right)_{8}\right]$ cluster $(\mathrm{M}=$ divalent metal ion, see Fig. 2(a)). ${ }^{55} \mathrm{~A}$ tetrazolate-based ligand with tetrahedral geometry, tetrakis(4-tetrazolylphenyl)methane $\left(\mathrm{H}_{4}\right.$ TTPM, see Section 1 in the ESI $\dagger$ ) should be utilized in this case. This framework, with a formula of $\mathrm{Cu}\left[\mathrm{Cu}_{4}\left(\mu_{4}-\mathrm{Cl}\right)(\mathrm{TTPM})_{2}\right]_{2}\left(\mathrm{CuCl}_{2}\right)(\mathrm{DMF})_{5}$ was prepared by a solvothermal reaction between $\mathrm{CuCl}_{2} \cdot \mathrm{H}_{2} \mathrm{O}$ and $\mathrm{H}_{4}$ TTPM in DMF-methanol mixture in the presence of $\mathrm{HCl}$ at room temperature for 4 days. Green tetragonal rod-like crystals were obtained from the reaction. This framework also possesses a fluorite topology net with an octahedral cavity size of $17.8 \times 17.8 \times 27.5 \AA$. (Fig. 2 (b)) Nevertheless, due to the anionic nature of its metal-containing SBUs, this 
(a)

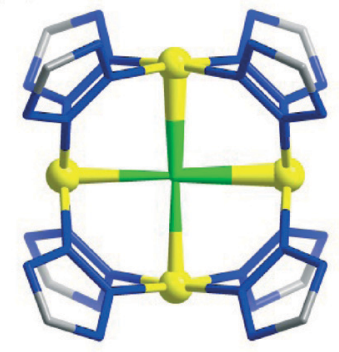

(b)

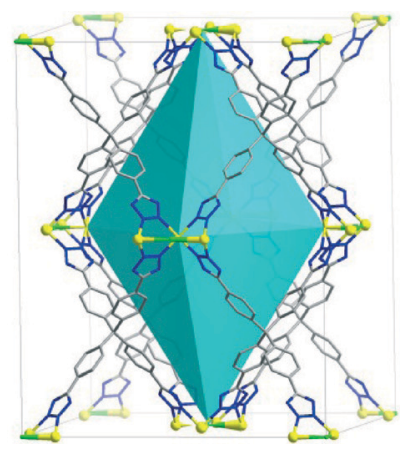

Fig. 2 (a) The 8-connected $\left.\left[\mathrm{Cu}_{4}\left(\mu_{4}-\mathrm{Cl}\right) \text { (tetrazolate) }\right)_{8}\right]$ cluster. (b) The graphic representation of another fluorite topology MOF with the tetrazolate ligand. The counterions are omitted for clarity. The turquoise polyhedron represents the octahedral interstitial cavities from the ccp of SBUs. Color scheme: C, gray; $\mathrm{N}$, blue; $\mathrm{Cl}$, green; and $\mathrm{Cu}$, yellow.

framework appears to be anionic where many bulky counterions were trapped in its octahedral holes. Its calculated solvent accessible volume is $19.70 \%$. This framework is porous upon activation, with a BET surface area of $2506 \mathrm{~m}^{2} \mathrm{~g}^{-1}$, while a structural change was observed during the activation process. ${ }^{56}$ An isostructural MOF, IMP-16 (IMP represents "Imperial College London"), which is made from an analogous silicon-centered tetrazolate ligand, has also recently been reported. It possesses a solvent accessible volume of $74.30 \%$ and a BET surface area of $2665 \mathrm{~m}^{2} \mathrm{~g}^{-1} .{ }^{57}$ It should be noted that a few other coordination polymers with fluorite topology ${ }^{35,45,58-65}$ have also been reported without conducting gas sorption measurements, presumably due to their small cavity sizes and instability of their frameworks.

2.1.2 MOFs with hierarchical cavity structures. Unlike the fluorite topology MOFs which have only one type of cavity (the interstitial octahedral cavities resulted from the cсp of the SBUs) with uniform size and shape, MOFs with other topologies may possess several different types of cavities. This makes it rather challenging to rationalize the shape and size of frameworks with these topologies. Utilization of metal-organic polyhedra (MOPs) or coordination polyhedra as building blocks appears to be an efficient strategy to construct highly-porous MOFs with hierarchical cavities. ${ }^{66,67}$ MOPs are supramolecular coordination assemblies of discrete cages that typically possess high symmetries, welldefined cavities and predictable structures. ${ }^{68-71}$ Isophthalates are one of the important categories of organic linkers for MOP construction. ${ }^{68}$ With a bridging angle of $120^{\circ}$ between the two adjacent carboxylates, the combination of 24 isophthalate moieties and 12 metal-containing SBUs with square planar geometry yields a cuboctahedral cage with $O_{\mathrm{h}}$ symmetry (Fig. 3(a)). ${ }^{68}$ So far, MOFs with cuboctahedral cages have been mostly explored due to the relatively easier synthesis of the $120^{\circ}$-angular-dicarboxylate ligands. A series of $(3,24)$ connected isoreticular MOFs with rht topology were constructed consequently using coplanar, $C_{3}$-symmetric ligands with three isophthalate moieties. ${ }^{7-78}$ Even though these MOFs are highly porous, their frameworks were
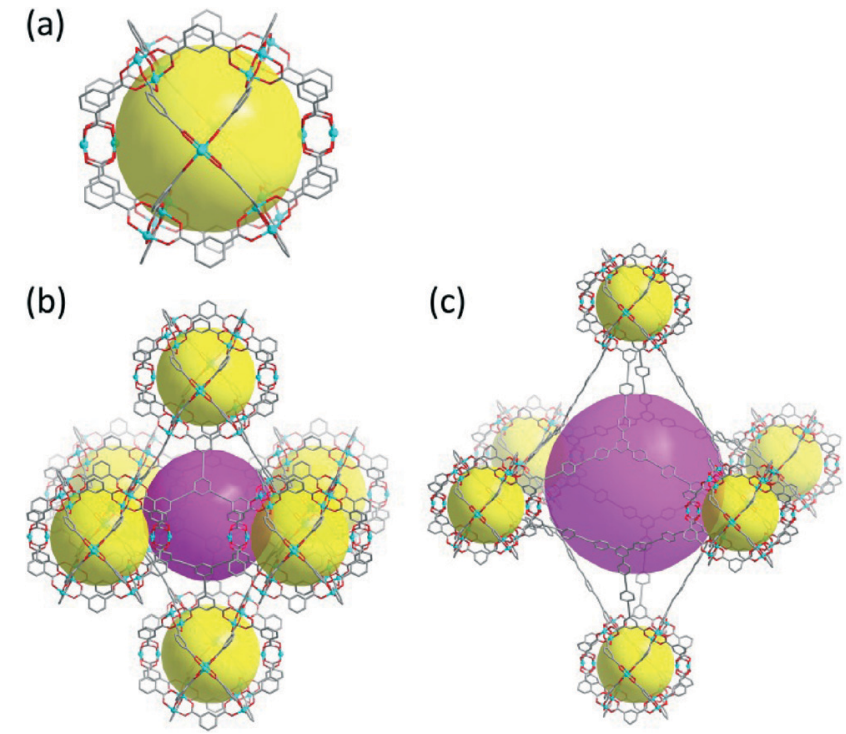

Fig. 3 (a) The graphic representation of a MOP with a cuboctahedral cage. The yellow sphere indicates the empty space inside its cage. (b, c) The crystal structures of PCN-61 and NU-110E, respectively. Both of their frameworks adopt the rht topology where their mesoporous cavities (illustrated as pink spheres) were stabilized by the introduction of the microporous cuboctahedral cages (illustrated as yellow spheres) made from the isophthalate moieties. Color scheme: C, gray; O, red; and $\mathrm{Cu}$, cyan.

stabilized by the incorporation of "microwindows" whose shape and size were fixed by the isophthalate moieties.

One of these $(3,24)$-connected isoreticular MOF series, PCN-61, ${ }^{72}$ was synthesized by a solvothermal reaction between $\mathrm{Cu}\left(\mathrm{NO}_{3}\right)_{2} \cdot 2.5 \mathrm{H}_{2} \mathrm{O}$ and $\mathrm{H}_{6} \mathrm{BTEI}\left(\mathrm{BTEI}=5,5^{\prime}, 5^{\prime \prime}\right.$-benzene-1,3,5-triyltris(1-ethynyl-2-isophthalate), see Section 1 in the ESI $\dagger$ ) in DMF in the presence of $\mathrm{HBF}_{4}$ at $75{ }^{\circ} \mathrm{C}$ for 3 days. Its crystal structure can be conceived as the packing of three different types of polyhedral cages with different sizes: a cuboctahedral cage with a diameter of $12 \AA$, a truncated tetrahedral cage with a diameter of $15 \AA$, and a truncated octahedral cage with a diameter of $23 \AA$. In this framework, its mesoporous cavities (the truncated octahedral cages, illustrated as pink spheres) were stabilized by the surrounding microporous cages (cuboctahedral cages, illustrated as yellow spheres) made from the isophthalate moieties (Fig. 3(b)). This framework possesses a solvent accessible volume of $77.0 \%$ and exhibits permanent inner porosity after the activation process, with a BET surface area of $3000 \mathrm{~m}^{2} \mathrm{~g}^{-1}$.

As the organic linker is elongated, the sizes of truncated tetrahedral cages and truncated octahedral cages will expand accordingly, while the size of cuboctahedral cages always remains constant. ${ }^{72,73}$ Consequently, the large cavity can always be stabilized by its surrounding microporous cuboctahedral cavities. Further elongation of the organic linker yields a series of highly porous MOFs, among which NU-110E (see Fig. 3(c), NU represents "Northwestern University") possesses the largest experimental BET surface area among all of the existing porous materials to date. ${ }^{78} \mathrm{NU}-110 \mathrm{E}$ consists of dicopper paddlewheel SBUs connected by the 
BTTEI ligand (BTTEI = 5, 5', $5^{\prime \prime}$-(((benzene-1,3,5-triyl-tris(ethyne2,1-diyl))tris(benzene-4,1-diyl)tris(ethyne-2,1-diyl))triisophthalate, see Section 1 in the ESI $\dagger$ ). This framework is isoreticular to the PCN-61 series. Due to its highly porous nature, supercritical $\mathrm{CO}_{2}$ activation has to be applied to maintain the integrity of its framework. It has a solvent accessible volume of $93.0 \%$ and exhibits a BET surface area of $7140 \mathrm{~m}^{2} \mathrm{~g}^{-1}$.

Successful construction of highly porous MOFs with rht topology has inspired chemists to continue the exploration of MOFs with other possible types of incorporated microscopic cavities. The octahedral cages that can be constructed from the $90^{\circ}$-angular-dicarboxylate ligands and square planar SBUs appear to be another interesting building block for highly porous MOFs. Due to the challenges in organic synthesis, this type of ligands still remains less explored. ${ }^{79,80} \mathrm{~A}$ dendritic compound, BTTCD (BTTCD = 9,9',9",9"' -([1,1'-biphenyl]3,3',5, $5^{\prime}$-tetrayl)tetrakis(9H-carbazole-3,6-dicarboxylate), see Section 1 in the ESI $\dagger$ ), featuring the $90^{\circ}$-bridging-angle between its two adjacent carboxylates, was investigated in this case. $\mathrm{PCN}-80^{79}$ was prepared by a solvothermal reaction between $\mathrm{Cu}\left(\mathrm{NO}_{3}\right)_{2} \cdot 2.5 \mathrm{H}_{2} \mathrm{O}$ and $\mathrm{H}_{8}$ BTTCD in DMF in the presence of $\mathrm{HBF}_{4}$. This framework consists of three distinctive types of microporous cavities packing in a $1: 1: 1$ ratio, with a large pore size of $16.6 \AA$, a middle pore size of $13.4 \AA$ and a small pore size of $7.6 \AA$. Similar to rht-topology MOFs, the large cavities were surrounded by a group of small cavities, whose shape and size were fixed by the bridging angle of adjacent carboxylates in its organic linkers (Fig. 4). This MOF has a solvent accessible volume of $72.1 \%$. It has a BET surface area of $3850 \mathrm{~m}^{2} \mathrm{~g}^{-1}$. Topologically, the combination of the BTTCD ligand that serves as a 3,3-connected node and the dicopper paddlewheel SBU gives rise to a unique topology of a $3,3,4$-connected net denoted as $\left(7^{2} \cdot 8^{2} \cdot 11^{2}\right)\left(7^{2} \cdot 8\right)\left(7^{3}\right)_{2}$. If the ligand topology was further simplified as an 8 -connected node, PCN-80 possesses a 4,8-connected net with scu topology. Due to its microporous nature and high density of unsaturated metal centers (UMCs), this MOF exhibits a $2.3 \mathrm{wt} \% \mathrm{H}_{2}$ uptake at $77 \mathrm{~K}$ and 1 bar, and an excess $\mathrm{H}_{2}$ uptake of $4.8 \mathrm{wt} \%$
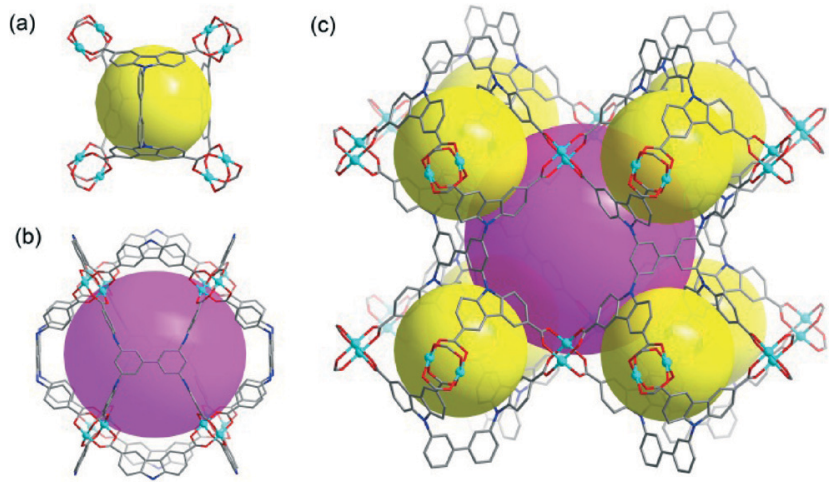

Fig. 4 (a, b) The graphic representation of the small and large cavities in $\mathrm{PCN}-80$. The yellow and pink spheres indicate the empty space inside the cavity. (c) The crystal structure of PCN-80, where the large cavities were stabilized by the neighboring small cavities. Color scheme: C, gray; O, red; $\mathrm{N}$, blue and $\mathrm{Cu}$, cyan. at $77 \mathrm{~K}$ and 44 bar, making it a promising material for hydrogen storage.

\subsection{Customizing the size and shape of MOF cavities for desired applications}

While controlling the framework topology is of vital significance for the rational design of MOFs with desired porosity and packing fashion, controlling the size and shape of each cavity in a framework allows researchers to synthesize materials purposefully built for a particular application. One of the applications for which the design of the cavity is of paramount importance is that of separation processes, such as separating $\mathrm{CO}_{2}$ from flue gas, or separating different hexane isomers. For these applications, the size and shape of the cavity directly influence the framework's adsorptive behavior to each molecule. For a given molecule, cavities with similar shape and size but complementary polarity will preferentially retain this particular molecule, and thus frameworks with these cavities will have much higher affinity to it. Our group published a few critical reviews on this topic ${ }^{8,9,12}$ several years ago, so here we seek to summarize recent progress from our group and other research groups.

2.2.1 MOFs with predesigned single molecular traps (SMTs) for $\mathrm{CO}_{2}$ capture. Even though much effort has been made to design frameworks with preferential adsorptive behaviors toward a general type of species, the construction of predesigned cavities that can accurately fit one single molecule is still largely challenging. Single molecular traps $(\mathrm{SMTs})^{81}$ were recently developed by our group, which are a simple coordination assembly of metal-containing units and organic linkers of the appropriate length and angle to form a cavity with predesigned size, shape and polarity. SMTs should show favorable and selective adsorption of the molecule that has the appropriate size and polarity to fit in (Fig. 5(a)). They can exist in both discrete cages and extended frameworks. In particular, a SMT cage can be functionalized with additional linking moieties to be incorporated into an extended MOF structure which contains accessible SMTs.

Our proof of concept for this technique involves the development of SMTs for $\mathrm{CO}_{2}$ capture. Due to climate change, efficient techniques for $\mathrm{CO}_{2}$ capture and sequestration are of great current interest for both researchers and the public. ${ }^{11,12}$ MOFs are one of the prime avenues of investigation towards this purpose. Due to their enormous surface area and convenient functionalization processes, they have the potential to adsorb and separate $\mathrm{CO}_{2}$ far more efficiently than traditional amine gas scrubbing technologies. ${ }^{82,83} \mathrm{CO}_{2}$ is a linear molecule with the size of approximately $2.4 \AA$, which has a partial negative charge at each oxygen atom and a partial positive charge on the central carbon atom. A pre-designed MOP as a SMT (Fig. 5(b)) was constructed using the ligand 3,3'-(naphthalene-2,7-diyl)dibenzoate (NDB, see Section 1 in the ESI $\dagger$ ) connected by dicopper paddlewheel SBUs. This cage, denoted as SMT-1, consists of two SBUs surrounded by four NDB ligands, making it a lantern-shaped molecular cage with a 

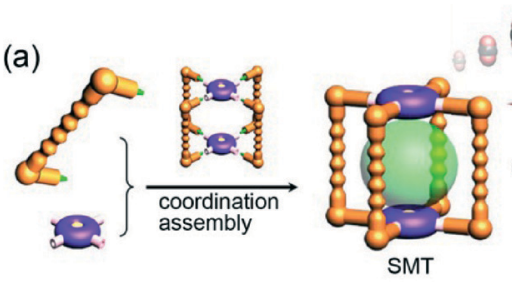

(b)

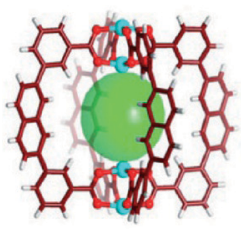

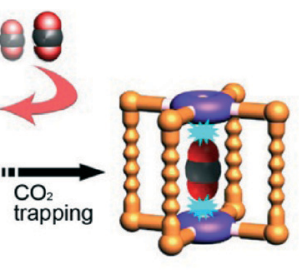

(d)

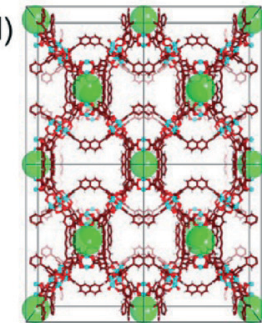

Fig. 5 (a) The illustration of the rational design of a SMT for $\mathrm{CO}_{2}$ capture. The organic linkers are shown in orange and the dimetal paddlewheel SBUs are shown in purple. (b) The single crystal structure of SMT-1. The green sphere indicates the pre-designed cavity to accommodate one $\mathrm{CO}_{2}$ molecule. (c) Extended 3D framework can be generated by functionalization of the ligand of SMT-1 with two extra carboxylates. (d) The single crystal structure of PCN-88, a SMTincorporated MOF. Color scheme: $\mathrm{C}$, brown; $\mathrm{O}$, red; $\mathrm{H}$, white, and $\mathrm{Cu}$, cyan. (Reprinted from ref. 81 with permission, copyright 2013 Nature Publishing Group.)

7.4 A gap between the two adjacent SBUs. This is a cage with exactly the right size to accommodate one $\mathrm{CO}_{2}$ molecule. In the as-synthesized form of SMT-1, each SBU is axially coordinated by two solvent molecules. These solvent molecules will be removed upon activation, which creates UMCs with a positive charge residing on each end of SMT. Through the customized electrostatic interaction between the negatively charged oxygen atom on $\mathrm{CO}_{2}$ and positively charged UMCs on the SMT, this SMT has exhibited a substantial preference for $\mathrm{CO}_{2}$ over any other gas molecules. ${ }^{81}$

After SMT-1 was shown to have promising $\mathrm{CO}_{2}$ selectivity as a MOP, it is highly desirable to incorporate the SMTs into an extended framework such as MOFs, which, in general, possess much larger surface areas and higher stabilities. By functionalizing the SMT-1 ligand with two additional carboxylates, a predesigned ligand for constructing SMT-incorporated MOFs, NDDA (NDDA = 5,5'-(naphthalene-2,7-diyl)diisophthalate, see Section 1 in the ESI $\dagger$ ) was investigated. Due to its additional carboxylates that are positioned at $120^{\circ}$ angles to the MOP carboxylates, it allows the formation of a three dimensional extended framework with many SMTs inherently incorporated in its structures (Fig. 5(c)). PCN-88 was prepared by a solvothermal reaction between $\mathrm{H}_{4} \mathrm{NDDA}$ and $\mathrm{Cu}\left(\mathrm{NO}_{3}\right)_{2} \cdot 2.5 \mathrm{H}_{2} \mathrm{O}$ in DMA at $85{ }^{\circ} \mathrm{C}$ for 2 days. This MOF contains two conformational isomers of the NDDA ligand. One isomer has a dihedral angle of nearly $90^{\circ}$ between the peripheral and central phenyl rings, and four of these ligands, combined with two dicopper paddlewheel SBUs, make up a unit identical to SMT-1 within the structure, in the shape of a quadrangular prism with a $7.4 \AA$ distance between the two UMCs, the right size for a $\mathrm{CO}_{2}$ molecule to fit in (Fig. 5(d)). The outward-facing carboxylates of this ligand link with eight other prisms through linking paddlewheels, creating other pores and forming the 3D framework. The other conformational isomer of NDDA adopts an almost coplanar conformation and is important in linking the SMTs and forming the larger pores. The activated sample of PCN- 88 shows a BET surface area of $3308 \mathrm{~m}^{2} \mathrm{~g}^{-1}$, as well as a significant $\mathrm{CO}_{2}$ uptake of $160 \mathrm{~cm}^{3} \mathrm{~g}^{-1}$ (and $94 \mathrm{~cm}^{3} \mathrm{~g}^{-1}$ ) at $273 \mathrm{~K}$ (and $296 \mathrm{~K}$ ) and $1 \mathrm{~atm}$. This is the highest value of $\mathrm{CO}_{2}$ uptake among all of the MOFs constructed from pure carboxylate ligands (without nitrogen functional groups). ${ }^{81}$ It should be noted that Mg-MOF-74, a MOF made from ligands with both hydroxyl and carboxylate moieties, has a higher $\mathrm{CO}_{2}$ uptake than PCN-88, presumably due to both the low atomic weight of magnesium and its high density of unsaturated magnesium centers. $^{84-88}$

This MOP-to-MOF design approach begins with the design of SMT cavities which are included into MOF structures and can provide customized adsorptive properties. These cavities must be designed with size, charge, and accessibility appropriate to host the desired molecules. PCN-88 contains both SMTs and larger pores. It is these larger pores that keep the SMTs accessible to the $\mathrm{CO}_{2}$ molecules and thus allow higher porosity of this framework. To prove that the selectivity was the result of using SMTs rather than simple adsorption of $\mathrm{CO}_{2}$ to the larger pores, $\mathrm{PCN}-88^{\prime}$ was synthesized under identical solvothermal conditions as PCN-88 in the presence of pyrazines. Single crystal structure analysis of PCN-88' shows that these independent pyrazine molecules have fit into the cage and blocked the SMTs, resulting in a considerably lower $\mathrm{CO}_{2}$ uptake at low pressure. ${ }^{81}$

It is suggested that the synthesis of a MOF whose entire cavities can serve as SMTs would further improve the $\mathrm{CO}_{2} / \mathrm{N}_{2}$ selectivity as compared to PCN-88. Similar to the SMTs in PCN-88, these "pockets" could be predesigned cavities that possess customized size and shape for preferential adsorption of a particular molecule (such as $\mathrm{CO}_{2}$ ). Our group recently explored the mixed ligand approach to produce a MOF with exclusive SMTs. In order to synthesize a MOF like this, two types of linkers were utilized, a rigid linker, tetrazolate-5-carboxylate (TZC, see Section 1 in the ESI $\dagger$ ), as a linker maintaining the MOF stability, and a flexible linker, 1,3-di(4-pyridyl)propane (DPP), which determines the size and shape of the pores. PCN-200 was synthesized by a hydrothermal reaction between sodium ethyl ester tetrazolate-5carboxylate (NaEtTZC), DPP and $\mathrm{Cu}(\mathrm{OAc})_{2} \cdot 2.5 \mathrm{H}_{2} \mathrm{O}$ in $\mathrm{H}_{2} \mathrm{O}$ at $150{ }^{\circ} \mathrm{C}$ for $24 \mathrm{~h} .{ }^{89}$ Single crystal X-ray diffraction reveals that the structure of PCN-200 possesses thin and rigid copper sheets formed from the linkage of the $\mu_{3}$-bridging TZC. The layers of copper sheets are separated by the flexible DPP ligands, which generates empty channels along the $c$ axis with a width of $4.4 \AA$ (Fig. 6(a)). Owing to the elastic nature of DPP ligands, these cavities can distort themselves based on the guest molecules they accommodate. The frameworkguest interaction can be monitored by synchrotron powder diffraction and Rietveld refinement. ${ }^{90}$ When the as-synthesized 
(a)

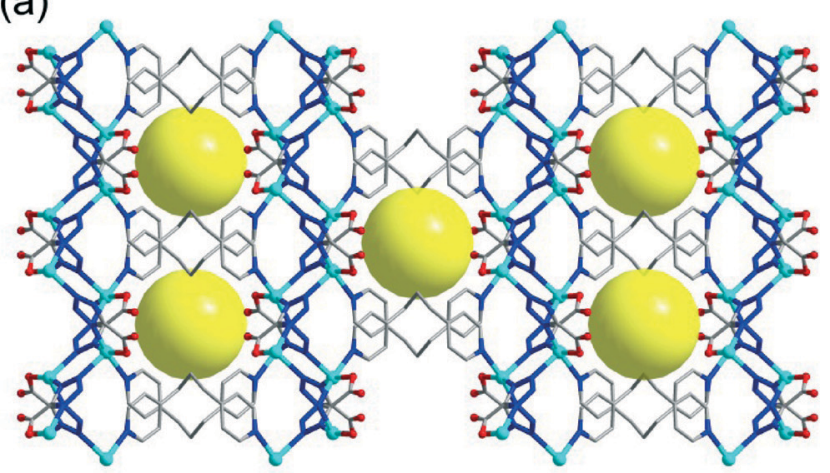

(b)

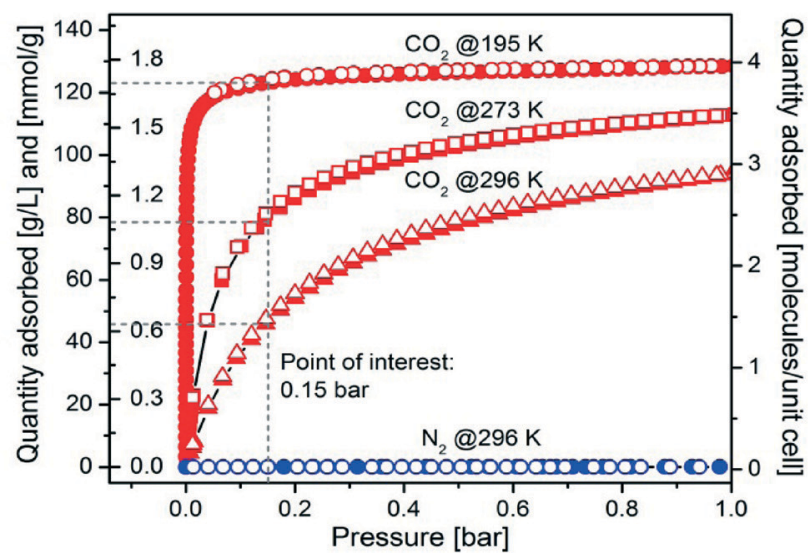

Fig. 6 (a) The single crystal structure of PCN-200. The yellow spheres indicate its open channels with the size of $4.4 \AA$ along the $c$ axis. Color scheme: C, gray; $\mathrm{O}$, red; $\mathrm{N}$, blue, and $\mathrm{Cu}$, cyan. (b) The adsorption isotherms of activated samples of PCN-200. $\mathrm{CO}_{2}$ at $195 \mathrm{~K}$ (red circles), $273 \mathrm{~K}$ (rectangles), and $296 \mathrm{~K}$ (triangles), and $\mathrm{N}_{2}$ at $296 \mathrm{~K}$ (blue circles). (Fig. 6(b) was reproduced with permission from ref. 89, copyright 2012 John Wiley \& Sons.)

PCN-200 material was activated, the water molecules that originally occupy the pores was removed and the overall structure changes were observed as evidenced by the unit cell angle $\beta$ changing from $92.657(10)^{\circ}$ to $116.087(4)^{\circ}$. In other words, it is the stimuli-responsive behavior observed in PCN-200 that has produced a polar pocket that selectively traps $\mathrm{CO}_{2}$ but excludes any other gas molecules, and thus resulted in its excellent $\mathrm{CO}_{2}$ selectivity. ${ }^{89}$

While PCN-200 does not have record-breaking $\mathrm{CO}_{2}$ working capacities, it shows nearly perfect selectivity for $\mathrm{CO}_{2}$ over $\mathrm{N}_{2}$ at room temperature, as expected for a structure wherein every cavity is an elastic SMT. At $195 \mathrm{~K}$ and 1 bar, a saturation of $\mathrm{CO}_{2}$ uptake in PCN-200 can be observed when four $\mathrm{CO}_{2}$ molecules were adsorbed in each unit cell. This implies a full occupation of $\mathrm{CO}_{2}$ in all existing cavities (Fig. 6(b)). At room temperature, this material retains $75 \%$ of its full $\mathrm{CO}_{2}$ capacity. Unlike any high-enthalpy adsorption interactions between $\mathrm{CO}_{2}$ and frameworks, the $\mathrm{CO}_{2}$ selectivity of PCN-200 is attributed to the presence of polar pockets with appropriate size to accommodate $\mathrm{CO}_{2}$; thus, PCN-200 requires significantly less energy to regenerate, but it still possesses an extremely high selectivity. Its water stability, easy activation process and the existence of elastic SMTs make it significantly more energy-efficient to produce a $\mathrm{CO}_{2}$ adsorptiondesorption cycle. ${ }^{89}$

2.2.2 MOFs with triangular channels for separation of hexane isomers. Low-energy purification and separation of liquid mixtures, at both the laboratory and industrial scales, are of tremendous interest to almost all chemists. Due to their large surface area, tunable pore size and adjustable surface functionalities, MOFs are promising materials that either can make new separation feasible or can make currently-performed separation processes more efficient. ${ }^{91}$ One example of a MOF with cavities designed for sizeselective separation of liquid has been recently reported by Long's group, which developed a MOF with the formula $\mathrm{Fe}_{2}(\mathrm{BDP})_{3}(\mathrm{BDP}=1,4$-benzenedipyrazole, see Section 1 in the $\mathrm{ESI}^{\dagger}$ ) for the separation of various isomers of hexane. This is an extremely important process for petroleum refinement since different hexane isomers are produced at an enormous scale through a catalytic isomerization reaction that results in a mixture of all five different isomers. ${ }^{92}$ This MOF consists of parallel linear chains of $\mathrm{Fe}^{3+}$ linked by BDP ligands (Fig. 7(a)), generating one-dimensional channels with a triangular shape (Fig. 7(b)). It is these triangular-shaped channels that give rise to the selective adsorption of hexane isomers (a)

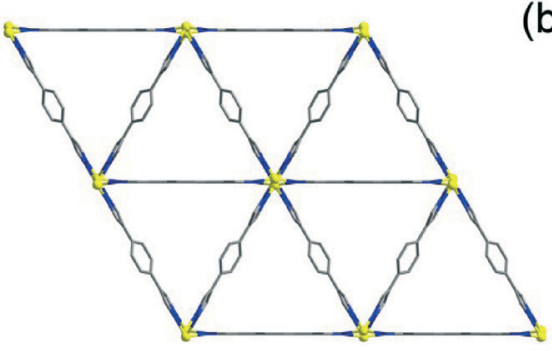

(c)

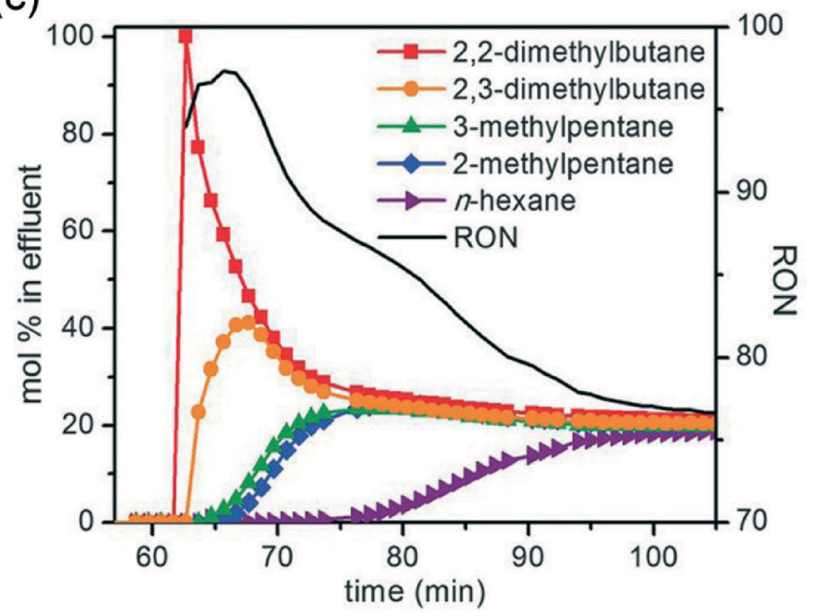

Fig. 7 (a) The single crystal structure of $\mathrm{Fe}_{2}(\mathrm{BDP})_{3}$ with its triangularshaped open channels. (b) The 1D chain of the pyrazolate-bridged $\mathrm{Fe}^{3+}$ octahedra. Color Scheme: C, gray; N, blue, and Fe, yellow. (c) The separation of an equimolar mixture of different hexane isomers. (Fig. 7(c) was reproduced with permission from ref. 92, copyright 2013 American Association for the Advancement of Science.) 
into this MOF. Isomers with a smaller degree of branching will have a stronger interaction with the channels. Unlike zeolites or many other MOF adsorbents which typically contain pores with obtuse angles, the cavities of $\mathrm{Fe}_{2}(\mathrm{BDP})_{3}$ possess acute crevices that are more accessible to linear alkanes than branched ones, resulting in an increased adsorption for the less branched molecules. The size of the triangular pores is also nearly ideal for this process. Consequently, the less branched isomers will have a longer retention time during the separation process (Fig. 7 (c)). The adsorption isotherms, breakthrough experiments, and configurational-bias Monte Carlo simulations all confirmed the more efficient separation of hexane isomer mixtures than found with current zeolites and previous MOFs. ${ }^{92}$

2.2.3 MOFs with mesoporous cavities induced by ligandfragment coassembly. Another important MOF application which requires accurate cavity design is heterogeneous catalysis. When designing catalytically active MOFs, several important factors should be considered. First, the size and shape of their pores or channels must be conducive to the diffusion of reactants and products. Second, these MOFs should be stable enough to endure harsh reaction conditions. Third, they should possess catalytic centers that are accessible to the reactants. It is a challenging problem to design a MOF that meets all of these requirements. Nevertheless, several methods, including the ligand-fragmentcoassembly strategy $^{93}$ and modulated synthesis strategy, ${ }^{94}$ have recently been developed for the purpose of simultaneous introduction of a certain degree of mesoporous cavities, UMCs and a variety of functional groups in a convenient manner. This enables us to combine the structural approach (designing larger cavities) and the functional approach (introducing UMCs and necessary functional groups) in the same MOF without employing complicated and expensive ligand synthesis. These studies show that it is possible for researchers to select a known structure with desired topology, stability and cost requirements out of the vast library of known MOFs, then quickly and cheaply introduce a tunable amount of mesoporous cavities or functional groups that they need for a particular application.

PCN-125 was designed through a ligand-fragmentcoassembly strategy. It is based on an existing structure, NOTT-101, which is an nbo-topology MOF with robustness and high $\mathrm{CO}_{2}$ and $\mathrm{H}_{2}$ uptake. ${ }^{95} \mathrm{PCN}-125$ was synthesized by a solvothermal reaction between $\mathrm{H}_{4}$ TPTC (TPTC = terphenyl$3,3^{\prime}, 5,5^{\prime}$-tetracarboxylate, see Scheme 2$)$ and $\mathrm{Cu}\left(\mathrm{NO}_{3}\right)_{2} \cdot 2.5 \mathrm{H}_{2} \mathrm{O}$ in DMA in the presence of a certain ratio of a truncated version of the ligand, that is, functionalized isophthalates. The resulting MOF retains the original structure of NOTT-101, where the existence of the fragment (functionalized isophthalates) in this framework has resulted in some defects in its crystal structure. It is these defects that have further enlarged the framework cavities and introduced various functional groups into the framework. In other words, the resulting MOF made from the ligand-fragment-coassembly strategy possesses mesoporous defects that contain whatever

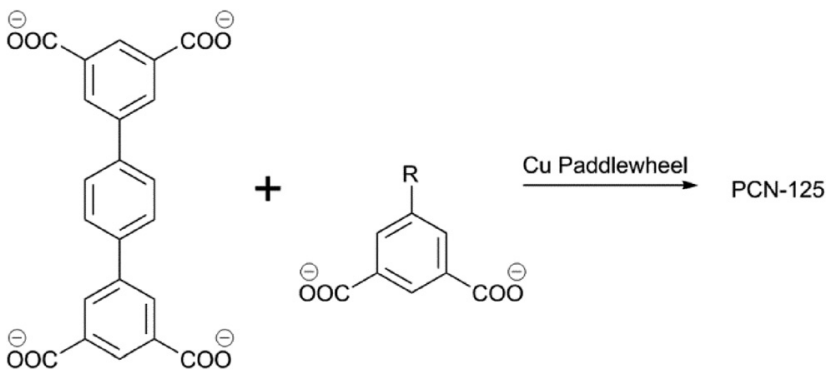

Ligand

Fragment

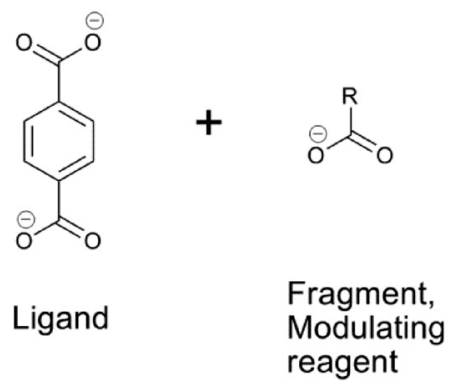

Scheme 2 The modulated synthesis strategy is an extensional work of the ligand-fragment-coassembly strategy on SBUs with a larger number of connectivity and greater stability. ( $\mathrm{R}=$ various functional groups.)

functional groups attached to the defect-creating truncated ligand (Scheme 2).

Traditional strategies of introducing functional groups into MOFs involve the functionalization of the original ligands (in this case, it is the TPTC ligand), which typically suffers from complicated organic syntheses and expensive reagents. However, due to the commercial availability and chemical accessibility of various isophthalates, the ligandfragment-coassembly has greatly simplified this process. In other words, it is significantly easier to functionalize an isopthalate molecule than a much more complex TPTC ligand. Furthermore, by varying the feed ratio of the TPTC ligand and the isopthalates, we can gain greater control of the porosity, the pore size distribution, and the degree of functionalization of this particular MOF. Studies have suggested that $\mathrm{PCN}-125$ could tolerate a very wide range of isophthalate feed ratios, from $4: 1$ to $1: 8$. This demonstrates that the concentration of the functional group in the MOF could be tuned by introducing a particular amount of the truncated ligand. Control of hierarchical micropores and mesopores in a wide variety of MOFs can now be investigated using this approach to improve mass transport. Additionally, we have demonstrated the synthesis of multifunctional MOFs by incorporating different isophthalate fragments in the same MOF, where adding multiple different functional groups to the same framework is a key step in the development of catalytically active MOFs.

Unlike the 4-connected dicopper paddlewheel SBUs in PCN-125/NOTT-101 which are susceptible to air and water, 
the 12-connected $\mathrm{Zr}_{6} \mathrm{O}_{8}$ clusters were demonstrated to possess greater chemical and thermal stability. It is suggested that the frameworks made from this SBU will have significantly improved stability and greater tolerance to defects, such as ligand fragments or UMCs. UiO-66 (see Fig. 8(a), UiO represents "University of Oslo") consists of the 12-connected $\mathrm{Zr}_{6} \mathrm{O}_{8}$ clusters (Fig. 8(b)) connected by BDC ligands $(\mathrm{BDC}=$ 1,4-benzenedicarboxylates, see Scheme 2). It was first discovered by Lillerud's group as polycrystalline powders. ${ }^{51}$ In order to improve the crystallinity of these zirconium-based MOFs, a modulated synthesis strategy was introduced by Behrens'94 and Kitagawa's ${ }^{96}$ groups who had prepared high-quality single crystals of $\mathrm{Zr}$ MOFs from solvothermal reactions in the presence of an excess amount of a monocarboxylic acid as a modulating reagent. ${ }^{25}$ It should be noted that a certain degree of defects will be introduced into the resulting $\mathrm{Zr}$ MOFs due to the presence of modulating reagents, in exactly the same manner as the mesoporous-defect-included PCN-125 made by the ligand-fragment-coassembly (Scheme 2).

In the defect-included UiO-66 structure, partial replacement of the BDC ligand with a non-connecting modulating reagent (a monocarboxylate) will result in the combination of adjacent pores, where mesoporous cavities can form in the structure (Fig. 8(d)). Removal of this non-connecting modulating ligand by various activation methods can generate unsaturated zirconium centers on the $\mathrm{Zr}_{6} \mathrm{O}_{8}$ clusters (Fig. 8(e)). Due to the large connectivity number of this cluster, removal of one linker will not sacrifice the overall (a)

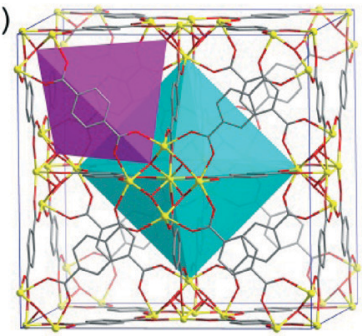

(d)

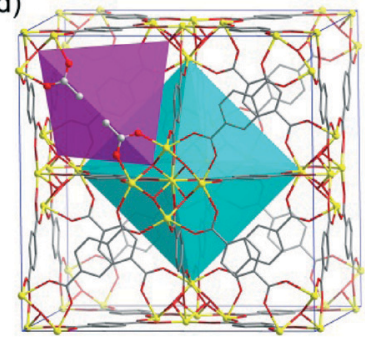

(b)

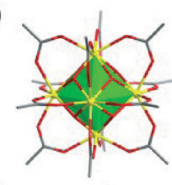

(c)

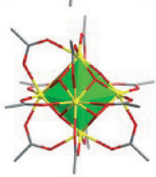

(e)

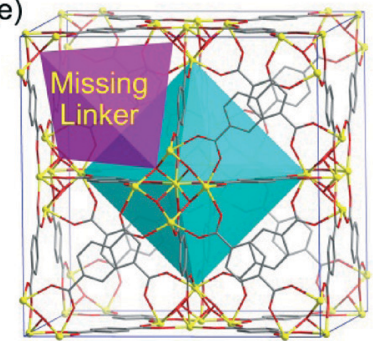

Fig. 8 The graphic illustration of the formation of defect-included UiO-66. (a) The crystal structure of UiO-66. The pink and cyan polyhedra represent its tetrahedral and octahedral cavities, respectively. (b) The normal 12-connected $\mathrm{Zr}_{6} \mathrm{O}_{8}$ cluster. The green polyhedra represent the octahedral geometry of the $\mathrm{Zr}_{6} \mathrm{O}_{8}$ cluster. (c) The 11connected $\mathrm{Zr}_{6} \mathrm{O}_{8}$ cluster with unsaturated $\mathrm{Zr}$ centers. (d) Defects can be introduced by replacing an original BDC linker with two modulating reagents (such as acetic acid in this case), which will result in a larger cavity by combining the two adjacent ones. (e) The removal of nonconnecting modulating reagents will result in both a larger cavity and unsaturated $\mathrm{Zr}$ centers. Color scheme: $\mathrm{C}$, gray; $\mathrm{O}$, red, and $\mathrm{Zr}$, yellow. stability of this framework (Fig. 8(c)). In fact, this strategy was simultaneously employed by several research groups, who prepared the defected-included UiO-66 using the modulated synthesis strategy, producing increased porosity (Fig. 9(a)) and enhanced functionality (Fig. 9(b)).

When UiO-66 was synthesized with acetic acid as the modulating reagent, W. Zhou and Yildirim's groups had confirmed the existence of the high concentration of these defects in UiO-66 and proved that these defects had increased the framework's porosity and gas adsorption. ${ }^{97}$ Fig. 9(a) shows that an increasing amount of $\mathrm{N}_{2}$ adsorption at $77 \mathrm{~K}$ was observed when UiO-66 was synthesized with an increasing volume of acetic acid. In addition, the isotherms display more type II behavior, which is evidence of increased mesoporosity. This increased mesoporosity without phase change was further substantiated by neutron powder diffraction measurements, finding a BDC occupancy of 89.0-93.8\%, depending on what hydroxyl or other groups might be left coordinating the $\mathrm{Zr}-\mathrm{O}$ units in the defects.

Concurrently, De Vos' group recently demonstrated the use of trifluoroacetic acid (TFA) to increase the catalytic activity of UiO-66 due to defect substitution of the ligand by the

(a)

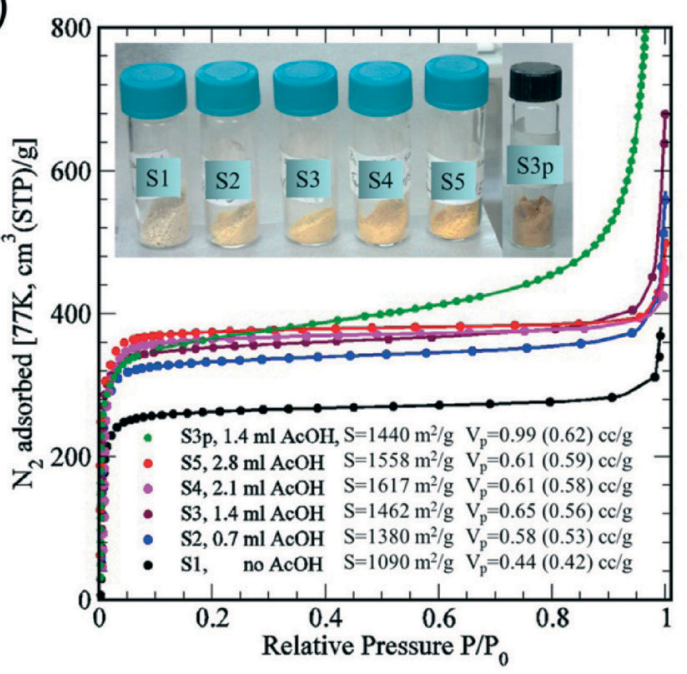

(b)
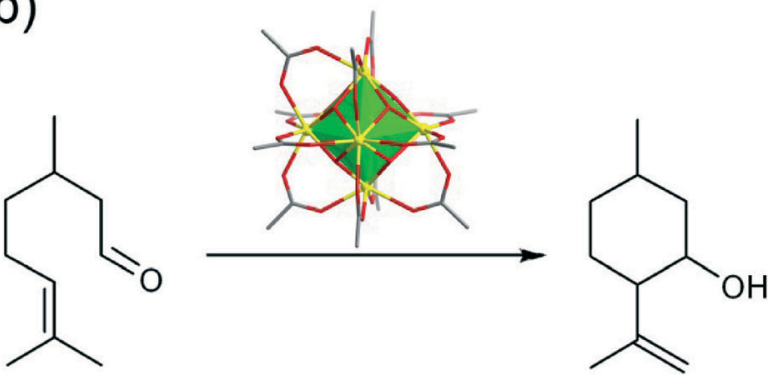

Fig. 9 (a) $\mathrm{N}_{2}$ adsorption isotherms of several UiO-66 samples synthesized using different amounts of acetic acid as the modulating reagent. (b) The catalytic reaction performed in the presence of UiO-66 synthesized using trifluoroacetic acid as the modulating reagent. (Fig. 9(a) was reproduced with permission from ref. 97, copyright 2013 American Chemical Society.) 
more labile TFA, followed by its removal during activation, joining adjacent cavities and creating more UMCs in the framework. ${ }^{98}$ This is also illustrated in Fig. 8(e) where two cavities that were normally separated by BDC linkers are joined after activation to form a larger cavity surrounded by two unsaturated zirconium centers. An increased UMC density was measured through $\mathrm{CD}_{3} \mathrm{CN}$ chemisorption followed by IR spectroscopy, showing an increase from $720 \mu \mathrm{mol} \mathrm{\textrm {g } ^ { - 1 }}$ Lewis acidic sites in unmodulated to $1100 \mu \mathrm{mol} \mathrm{g}^{-1}$ in TFAmodulated UiO-66 samples. The existence of UMCs has greatly improved the catalytic activity of this framework, such as the conversion of citronellal to isopulegol (Fig. 9(b)). These two studies independently and unambiguously confirmed the presence of linker vacancies in UiO-66 after a modulating reagent was used in the synthesis and that the number of defects can be tuned through altering the amount of the modulator.

\section{Rational design of MOFs with anticipated functionalities}

While the utilization of different metal-containing SBUs and organic linkers generates a great variety of MOF structures, introduction of various functionalities into MOFs will provide MOFs a wide range of properties and applications. It appears that without functionalization, the application of MOFs would be severely limited. The presence of various functional groups will significantly enrich the usefulness of MOFs. Many different functional groups, such as diazo groups, Lewis bases, UMCs, and metalloporphyrin centers could be incorporated into MOFs for the purpose of constructing functional MOFs with enhanced performance in optical sensitivity or catalytic activity. Herein we would like to present various ways of introducing expected functionalities into MOF materials.

\subsection{Synthesis of optically sensitive MOFs via pre-synthetic ligand design}

The use of external stimuli to control the adsorptivity of MOFs has been a topic that has garnered a lot of attention in recent years. The ability to control the adsorptive behaviors of a material with the figurative "flip of a switch" allows for simple capture and release of a substrate. MOFs are promising candidates for tunable adsorptivity via ligand manipulation because of their large pores and well defined structures in which functionalized ligands can be readily incorporated. One example of this controlled adsorptivity can be found in $\mathrm{PCN}-123,{ }^{99}$ which was synthesized via a solvothermal reaction between $\mathrm{Zn}\left(\mathrm{NO}_{3}\right)_{2} \cdot 6 \mathrm{H}_{2} \mathrm{O}$ and $\mathrm{H}_{2} \mathrm{PDT}$ (PDT = 2-(phenyldiazenyl)terephthalate see Section 1 in the ESI $\dagger$ ) in $\mathrm{DEF}$ at $85^{\circ} \mathrm{C}$ for 2 days. Bearing the optically sensitive diazo group, the PDT ligand can undergo photochemical isomerization when exposed to UV radiation or heat, as seen in Fig. 10. Ligand isomerization changes the shape, size and polarity of the pores, thus allowing optical and thermal control of the adsorptive behavior of this material. Before any exposure to

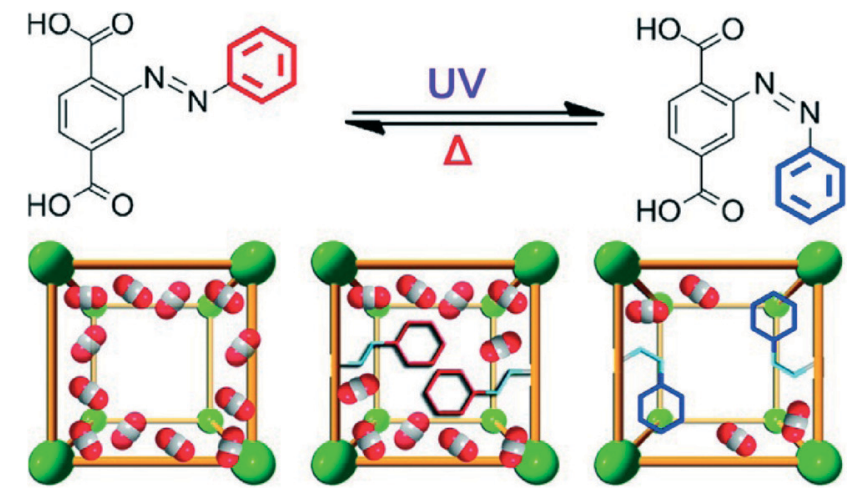

Fig. 10 Photochemical isomerization of the PDT ligand induced by UV radiation or heat can result in controlled adsorptivity of $\mathrm{CO}_{2}$ in PCN-125. (Reprinted from ref. 99 with permission, copyright 2012 American Chemical Society.)

UV radiation, $\mathrm{PCN}-123$ has a $\mathrm{CO}_{2}$ uptake of $22.9 \mathrm{~cm}^{3} \mathrm{~g}^{-1}$ at 1 bar, but that uptake decreases to $10.5 \mathrm{~cm}^{3} \mathrm{~g}^{-1}$ after 1 hour of UV exposure, a decrease of $53.9 \%$. Taking the irradiated PCN-123 samples and heating them at $60{ }^{\circ} \mathrm{C}$ for 20 hours increase the adsorption to $26.4 \mathrm{~cm}^{3} \mathrm{~g}^{-1}$ at $1 \mathrm{bar}$, an increase of $13.3 \%$ over the initial adsorption measured. A pristine sample of PCN-123 starts predominantly with the trans ligand and some cis ligand, and UV radiation isomerizes the ligand into the cis form, as seen in Fig. 10. Heating the sample then isomerizes the cis ligand into the trans form, resulting in the increase in $\mathrm{CO}_{2}$ adsorption. In addition, $\mathrm{PCN}-123$ can go through multiple cycles of UV exposure and heating and still maintain its tunable adsorptivity. After the second UV exposure, the $\mathrm{CO}_{2}$ uptake decreased to $14.2 \mathrm{~cm}^{3} \mathrm{~g}^{-1}$. When heated again, the uptake increased to $22.4 \mathrm{~cm}^{3} \mathrm{~g}^{-1}$. This shows that PCN-123 is a viable material for controlled storage and release of $\mathrm{CO}_{2}$ and possibly other similar substrates.

A similar MOF, azo-IRMOF-74-III, ${ }^{100}$ has been reported by Stoddart and Yaghi's groups. This MOF is synthesized by a solvothermal reaction between $\mathrm{Mg}\left(\mathrm{NO}_{3}\right)_{2} \cdot 6 \mathrm{H}_{2} \mathrm{O}$ and the azobenzene-functionalized ligand in a DMF-ethanol- $\mathrm{H}_{2} \mathrm{O}$ mixture at $120{ }^{\circ} \mathrm{C}$ for $24 \mathrm{~h}$. It has a BET surface area of $2410 \mathrm{~m}^{2} \mathrm{~g}^{-1}$, which is, so far, the highest surface area among the MOFs containing photoswitchable modules. The inspiration for this MOF was to create an isoreticular expansion of MOF-74 whose adsorptivity can also be easily controlled by external stimuli. Unlike $\mathrm{PCN}-123$ where the isomer of the diazo groups suffers from the steric hindrance of the square pores, the steric hindrance in azo-IRMOF-74-III was greatly reduced by the large non-interpenetrated 1-D hexagonal pores. Photochemical isomerization can significantly change the pore size of this MOF. When the diazo group was in the trans configuration, the pore size was $0.83 \mathrm{~nm}$ and when it was converted to the cis configuration, the size increased to $1.03 \mathrm{~nm}$.

\subsection{MOFs for heterogeneous catalysis}

In addition to MOFs with photoswitchable properties, heterogeneous catalysts are also an important application of MOFs 
where ligand design is of vital importance. MOFs are excellent candidates as catalysts because it is convenient to incorporate and distribute different catalytic centers into the structure. MOFs have an advantage over homogeneous catalysts because of the convenient loading and separation process from the reaction mixture. Herein, a few representatives of catalytically active MOFs made from pre-synthetic ligand design will be discussed in this section.

3.2.1 Synthesis of MOFs for tandem catalysis. PCN-124 is a MOF that contains both Lewis acid and Lewis base moieties that can cooperatively catalyze a tandem reaction. This MOF was synthesized by a solvothermal reaction between $\mathrm{H}_{4} \mathrm{PDAI}$ (PDAI = 5,5'-((pyridine-3,5-dicarbonyl)bis(azanediyl))diisophthalate, see Fig. 11(a)) and $\mathrm{Cu}\left(\mathrm{NO}_{3}\right)_{2} \cdot 2.5 \mathrm{H}_{2} \mathrm{O}$ in DMA- $\mathrm{H}_{2} \mathrm{O}$ mixture. In this MOF the organic linker acts as a Lewis base while the unsaturated copper centers on the dicopper paddlewheel SBU (Fig. 11(b)) act as Lewis acids. ${ }^{101}$ Similar to PCN-6X ${ }^{72,73}$ / NU-110 ${ }^{78}$ series, PCN-125 also consists of cuboctahedral cages made from twelve dicopper paddlewheel SBUs and twenty-four isophthalate moieties (Fig. 3(a)). These cages are connected by the ligands to form a framework with cubic symmetry (Fig. 11(c)). Due to large pore sizes and one of the axial sites of the copper paddlewheel coordinating with the pyridine group on the ligand, the actual framework of PCN-124 is a doubly-interpenetrated structure (Fig. 11(d)).

PCN-124 was successfully used as a heterogeneous catalyst for the deacetalization-Knoevenagel condensation. The first step of the reaction is the catalyzed deacetalization of dimethoxymethylbenzene to form benzaldehyde. The second step converts benzaldehyde to benzylidene malononitrile. Benzylidene malononitrile can be used as an inhibitor for protein tyrosine kinase via the Knoevenagel reaction. Usually the Knoevenagel reaction is catalyzed by a base, but the
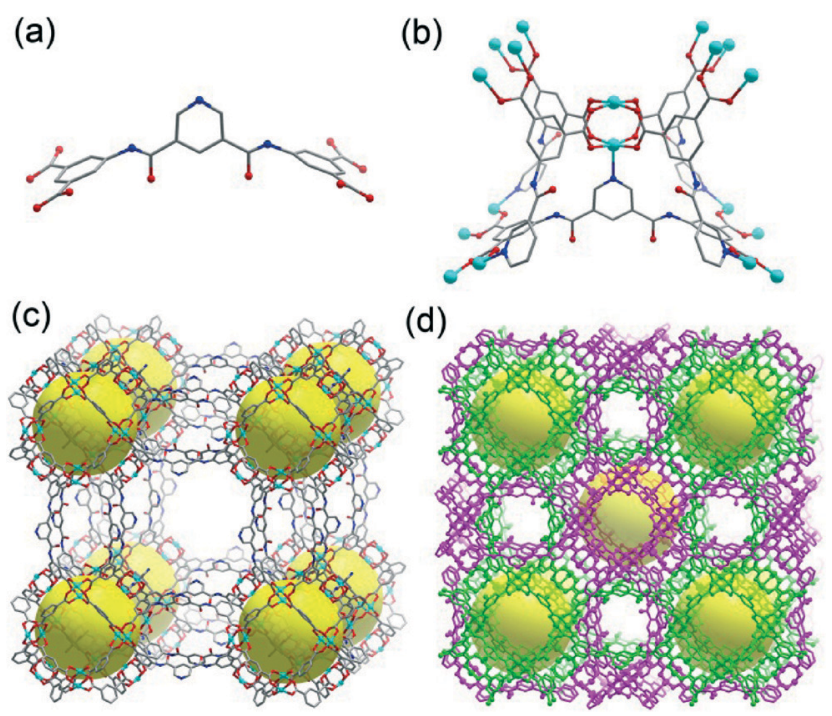

Fig. 11 (a) The structure of the PDAI ligand. (b) The coordination environment of the dicopper paddlewheel SBU. (c) A single cubic framework in PCN-124. (d) The self-interpenetrated structure of PCN-124. Color scheme: C, gray; O, red; N, blue, and Cu, cyan. The yellow sphere indicates the empty space inside its cuboctahedral cage. amides and pyridines in PCN-124 act as bases, replacing the need for additional bases. PCN-124 proved to be a viable catalyst with a turnover number (TON) of about 190. In addition, PCN-124 is easily recoverable after the reaction. Simply centrifuging the reaction vial separates the MOF from the rest of the solution.

In addition to being an excellent catalyst, PCN-124 also exhibits high $\mathrm{CO}_{2}$ selectivity. $\mathrm{CO}_{2}$ uptake in PCN-124 was found to be as high as $204 \mathrm{~cm}^{3} \mathrm{~g}^{-1}$ at $273 \mathrm{~K}$ and $760 \mathrm{mmHg}$. In comparison, $\mathrm{CH}_{4}$ uptake was $33.4 \mathrm{~cm}^{3} \mathrm{~g}^{-1}$ under the same conditions. This large difference in adsorption shows that PCN-124 is also a promising material for $\mathrm{CO}_{2} / \mathrm{CH}_{4}$ separation.

3.2.2 Synthesis of zirconium MOFs for biomimetic catalysis. Regardless of the versatile applications mentioned, one major obstacle of the majority of MOFs is their general instability toward water, air, and other harsh conditions. For example, PCN-124 is relatively unstable due to its copper SBUs. In order for MOFs to provide economical functionality, high-valence metals should be employed to improve the framework stability. ${ }^{25}$ Zirconium polyoxo clusters have attracted our attention due to their tunable connectivities (Fig. 12). So far, 12-connected, ${ }^{51,94}$ 8-connected ${ }^{25,36,102}$ and 6-connected $\mathrm{Zr}_{6} \mathrm{O}_{8}$ clusters ${ }^{103}$ were identified in MOFs, and all of them have been demonstrated to be air and moisture stable.

Drawing inspiration from nature, our group has recently synthesized a variety of ultra-stable porphyrin MOFs for use as catalysts and gas storage materials. Metalloporphyrins can be found in nature as light harvesters, oxygen transporters, and catalysts. ${ }^{102}$ Incorporation of metalloporphyrins into MOFs will significantly enrich the MOF functionalities. Using $\mathrm{Zr}$ clusters as SBUs, PCN-222 was synthesized via a solvothermal reaction between Fe-TCCP (TCCP $=$ tetrakis(4-
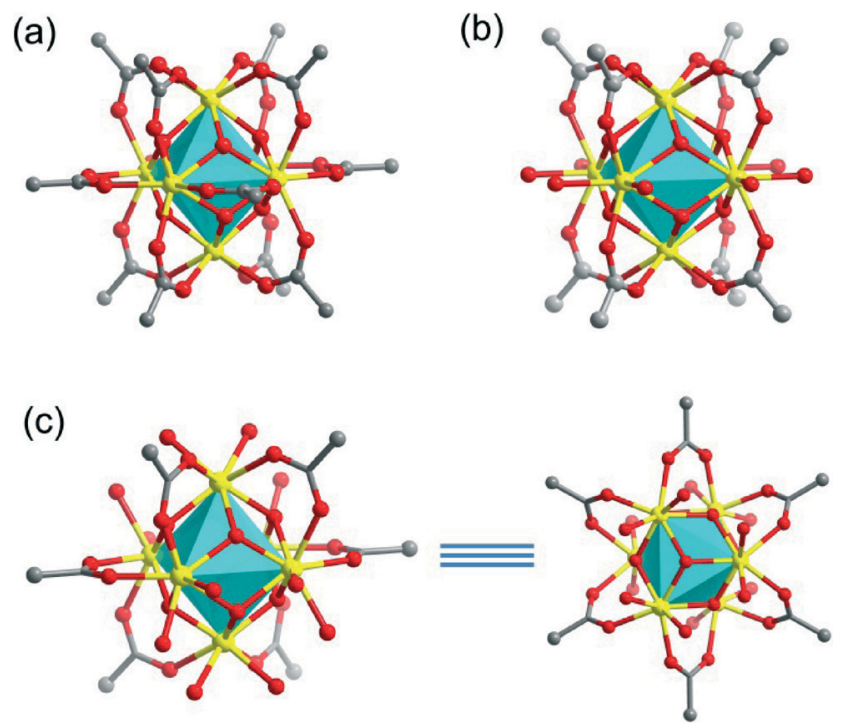

Fig. 12 Different connectivities of $\mathrm{Zr}_{6} \mathrm{O}_{8}$ clusters: (a) 12-connected; (b) 8-connected; (c) 6-connected (left: side view; right: top view). Color scheme: $\mathrm{C}$, gray; $\mathrm{O}$, red, and $\mathrm{Zr}$, yellow. The cyan polyhedra represent the octahedral geometry of the $\mathrm{Zr}_{6} \mathrm{O}_{8}$ cluster. 
carboxyphenyl)porphyrin, see Fig. 13) with $\mathrm{ZrCl}_{4}$ in DMF or DEF in the presence of benzoic acid. As seen in Fig. 13, each Fe-TCCP ligand is attached to four of the 8-connected $\mathrm{Zr}_{6} \mathrm{O}_{8}$ clusters with a twisted angle to form a $3 \mathrm{D}$ network with a Kagome-like topology. PCN-222 has two different channels, mesoporous hexagonal channels with diameters as large as $3.7 \mathrm{~nm}$ and microporous triangular channels with a diameter of $1.3 \mathrm{~nm}$. It has a $\mathrm{N}_{2}$ uptake of $1009 \mathrm{~cm}^{3} \mathrm{~g}^{-1}$ and a BET surface area of $2200 \mathrm{~m}^{2} \mathrm{~g}^{-1}$. In addition to its large pore size, PCN-222 is incredibly stable, maintaining its framework integrity not only in water, but in boiling water and concentrated hydrochloric acid. These attributes make it an excellent candidate for use as a biomimetic catalyst. Kinetic studies have shown that PCN-222 makes for an excellent peroxidase-like catalyst. PCN-222 was used to oxidize several substrates, including pyrogallol, 3,3,5,5-tetramethylbenzidine, and $o$-phenylenediamine (Scheme 3(a)). PCN-222 was found to have $k_{\text {cat }}$ of $16.1,14.0$, and $7.3 \mathrm{~min}^{-1}$, respectively, for these reactions. These values are several times higher than those of other catalysts such as hemin. ${ }^{104}$

By simply adjusting the reaction conditions of PCN-222, a new MOF, PCN-224, ${ }^{103}$ was identified as a metalloporphyrinfunctionalized MOF that consists of TCPP ligands with $\mathrm{Zr}_{6} \mathrm{O}_{8}$ clusters. The difference between PCN-222 and PCN-224 comes from having 6-connected $\mathrm{Zr}_{6} \mathrm{O}_{8}$ clusters in PCN-224 as opposed to 8-connected clusters in PCN-222, as seen in Fig. 13. This results in the formation of square channels with

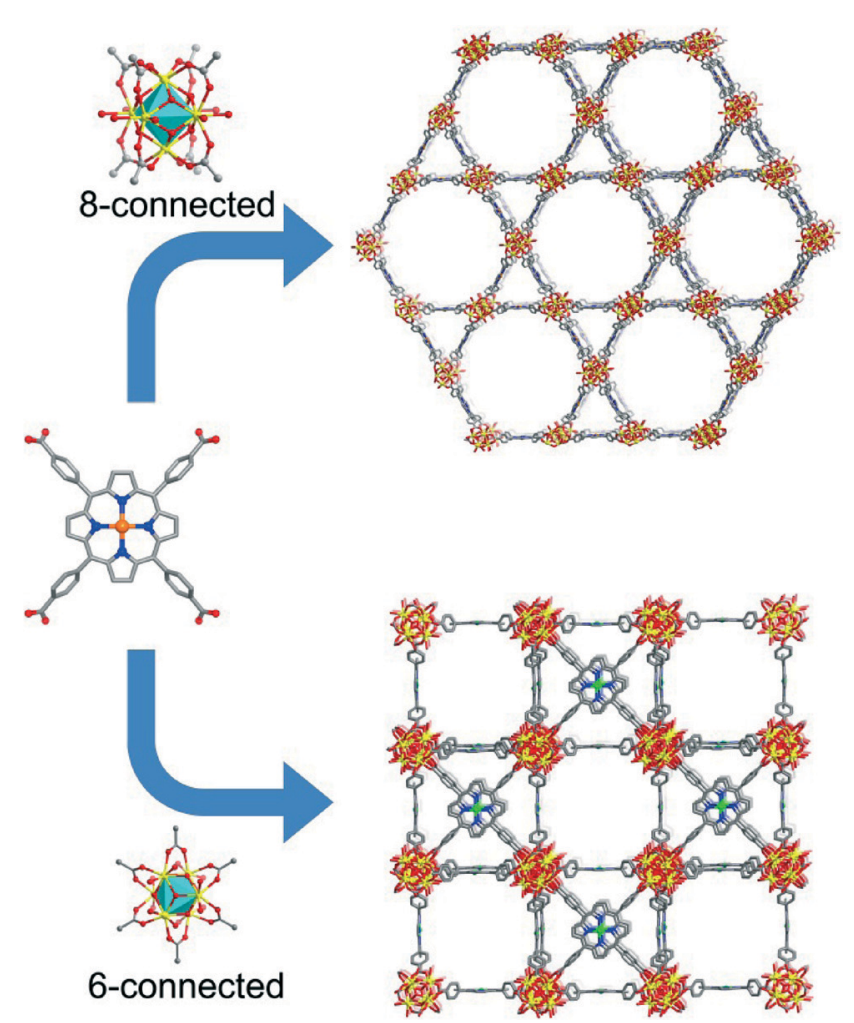

Fig. 13 The ligands, SBUs and crystallographic structures of PCN-222 (top) and PCN-224 (bottom). Color scheme: C, gray; O, red; N, blue, and $\mathrm{Zr}$, yellow. (a)<smiles>O=c1c(O)cccc2cc(O)c(O)c(O)c12</smiles>

(b)

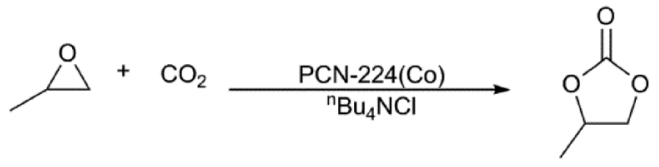

Scheme 3 (a) The biomimetic reaction catalyzed by PCN-222 (b) The $\mathrm{CO}_{2}$ /epoxide coupling reaction catalyzed by $\mathrm{PCN}-224$

a diameter of $1.9 \mathrm{~nm}$ in the structure of PCN-224. Like PCN-222, PCN-224 shows ultra-high stability in solutions ranging from $\mathrm{pH}=0$ to $\mathrm{pH}=11$, a great improvement over PCN-222 which is not stable under basic conditions. In addition to its stability, PCN-224 showed $\mathrm{N}_{2}$ uptake of $790 \mathrm{~cm}^{3} \mathrm{~g}^{-1}$ and a BET surface area of $2600 \mathrm{~m}^{2} \mathrm{~g}^{-1}$, one of the highest surface areas reported for porphyrin MOFs.

Just as PCN-222 showed promise as a biomimetic catalyst, PCN-224 proved to be an excellent catalyst for $\mathrm{CO}_{2} /$ epoxide coupling (Scheme 3(b)) with a turnover frequency (TOF) of around 120. Normally $\mathrm{CO}_{2}$ and epoxide coupling requires high pressures of $\mathrm{CO}_{2}$ to maintain enough dissolved $\mathrm{CO}_{2}$ in solution. But with PCN-224, one can preload the MOF with $\mathrm{CO}_{2}$ and use it as a heterogeneous catalyst which steadily releases $\mathrm{CO}_{2}$ into the reaction system.

\subsection{Post-synthetic modification of MOFs}

In addition to the pre-synthetic ligand design, post-synthetic modifications (PSMs) appear to be another important way to introduce a great variety of functional groups into the MOF framework. PSM is a process in which the material is chemically modified without damaging the integrity or changing the infrastructure of its framework. In consideration of several comprehensive reviews that have been recently published on this topic, ${ }^{31,32}$ this section will not be discussed in detail to avoid redundancy.

\section{Conclusions}

It is highly desirable to rationally design and synthesize materials with anticipated performance. In this highlight review, we have covered important recent advances on this topic. Two different approaches of rational MOF design, a structural approach and a functional approach, were elaborated. By implementation of the symmetry-guided design of MOFs with desired topology, MOFs with ultrahigh surface area can be obtained. Incorporation of pre-designed building blocks or cavities into the MOF structure can generate MOFs with desired adsorptive behavior. Utilization of pre-synthetically designed organic linkers and employment of the post-synthetic modification strategy are efficient ways to construct MOFs with desired functional groups and expected properties. This 
work also offers a general perspective of rational design of MOFs with expected porosities and functionalities.

\section{Acknowledgements}

This work was supported as part of the Center for Gas Separations Relevant to Clean Energy Technologies, an Energy Frontier Research Center funded by the U.S. Department of Energy (DOE), the Office of Science and the Office of Basic Energy Sciences under award number DE-SC0001015, part of the Hydrogen and Fuel Cell Program under award number DE-FC36-07G017033, part of the Methane Opportunities for Vehicular Energy (MOVE) Program, an ARPA-e project under award number DE-AR0000249 and part of the Welch Foundation under award number A-1725. M. B. also acknowledges the Texas A\&M graduate merit fellowship.

\section{Notes and references}

1 H.-C. Zhou, J. R. Long and O. M. Yaghi, Chem. Rev., 2012, 112, 673-674.

2 J. R. Long and O. M. Yaghi, Chem. Soc. Rev., 2009, 38, 1213-1214.

3 S. R. Batten, N. R. Champness, X.-M. Chen, J. Garcia-Martinez, S. Kitagawa, L. Öhrström, O. K. Michael, M. P. Suh and J. Reedijk, Pure Appl. Chem., 2013, 85, 1715-1724.

4 J. Sculley, D. Yuan and H.-C. Zhou, Energy Environ. Sci., 2011, 4, 2721-2735.

5 M. P. Suh, H. J. Park, T. K. Prasad and D.-W. Lim, Chem. Rev., 2011, 112, 782-835.

6 T. A. Makal, J.-R. Li, W. Lu and H.-C. Zhou, Chem. Soc. Rev., 2012, 41, 7761-7779.

7 E. D. Bloch, W. L. Queen, R. Krishna, J. M. Zadrozny, C. M. Brown and J. R. Long, Science, 2012, 335, 1606-1610.

8 J.-R. Li, R. J. Kuppler and H.-C. Zhou, Chem. Soc. Rev., 2009, 38, 1477-1504.

9 J.-R. Li, J. Sculley and H.-C. Zhou, Chem. Rev., 2011, 112, 869-932.

10 T. M. McDonald, W. R. Lee, J. A. Mason, B. M. Wiers, C. S. Hong and J. R. Long, J. Am. Chem. Soc., 2012, 134, 7056-7065.

11 K. Sumida, D. L. Rogow, J. A. Mason, T. M. McDonald, E. D. Bloch, Z. R. Herm, T.-H. Bae and J. R. Long, Chem. Rev., 2011, 112, 724-781.

12 J.-R. Li, Y. Ma, M. C. McCarthy, J. Sculley, J. Yu, H.-K. Jeong, P. B. Balbuena and H.-C. Zhou, Coord. Chem. Rev., 2011, 255, 1791-1823.

13 L. Ma, C. Abney and W. Lin, Chem. Soc. Rev., 2009, 38, 1248-1256.

14 M. Yoon, R. Srirambalaji and K. Kim, Chem. Rev., 2011, 112, 1196-1231.

15 M. D. Allendorf, C. A. Bauer, R. K. Bhakta and R. J. T. Houk, Chem. Soc. Rev., 2009, 38, 1330-1352.

16 Y. Cui, Y. Yue, G. Qian and B. Chen, Chem. Rev., 2011, 112, 1126-1162.
17 P. Horcajada, T. Chalati, C. Serre, B. Gillet, C. Sebrie, T. Baati, J. F. Eubank, D. Heurtaux, P. Clayette, C. Kreuz, J.-S. Chang, Y. K. Hwang, V. Marsaud, P.-N. Bories, L. Cynober, S. Gil, G. Ferey, P. Couvreur and R. Gref, Nat. Mater., 2010, 9, 172-178.

18 P. Horcajada, R. Gref, T. Baati, P. K. Allan, G. Maurin, P. Couvreur, G. Férey, R. E. Morris and C. Serre, Chem. Rev., 2011, 112, 1232-1268.

19 H. Li, M. Eddaoudi, M. O'Keeffe and O. M. Yaghi, Nature, 1999, 402, 276-279.

20 S. S.-Y. Chui, S. M.-F. Lo, J. P. H. Charmant, A. G. Orpen and I. D. Williams, Science, 1999, 283, 1148-1150.

21 H. Furukawa, K. E. Cordova, M. O'Keeffe and O. M. Yaghi, Science, 2013, 341, 1230444.

22 S. M. Kuznicki, V. A. Bell, S. Nair, H. W. Hillhouse, R. M. Jacubinas, C. M. Braunbarth, B. H. Toby and M. Tsapatsis, Nature, 2001, 412, 720-724.

23 B. Chen, S. Ma, F. Zapata, F. R. Fronczek, E. B. Lobkovsky and H.-C. Zhou, Inorg. Chem., 2007, 46, 1233-1236.

24 O. K. Farha and J. T. Hupp, Acc. Chem. Res., 2010, 43, 1166-1175.

25 M. Zhang, Y.-P. Chen, M. Bosch, T. Gentle, K. Wang, D. Feng, Z. U. Wang and H.-C. Zhou, Angew. Chem., Int. Ed., 2014, 53, 815-818.

26 M. Wriedt, A. A. Yakovenko, G. J. Halder, A. V. Prosvirin, K. R. Dunbar and H.-C. Zhou, J. Am. Chem. Soc., 2013, 135, 4040-4050.

27 M. Eddaoudi, J. Kim, N. Rosi, D. Vodak, J. Wachter, M. O'Keeffe and O. M. Yaghi, Science, 2002, 295, 469-472.

28 O. M. Yaghi, M. O'Keeffe, N. W. Ockwig, H. K. Chae, M. Eddaoudi and J. Kim, Nature, 2003, 423, 705-714.

29 D. Zhao, D. J. Timmons, D. Yuan and H.-C. Zhou, Acc. Chem. Res., 2010, 44, 123-133.

30 F. A. Almeida Paz, J. Klinowski, S. M. F. Vilela, J. P. C. Tome, J. A. S. Cavaleiro and J. Rocha, Chem. Soc. Rev., 2012, 41, 1088-1110.

31 Z. Wang and S. M. Cohen, Chem. Soc. Rev., 2009, 38, 1315-1329.

32 S. M. Cohen, Chem. Rev., 2011, 112, 970-1000.

33 X.-S. Wang, S. Ma, P. M. Forster, D. Yuan, J. Eckert, J. J. López, B. J. Murphy, J. B. Parise and H.-C. Zhou, Angew. Chem., Int. Ed., 2008, 47, 7263-7266.

34 D. Sun, Y. Ke, T. M. Mattox, B. A. Ooro and H.-C. Zhou, Chem. Commun., 2005, 5447-5449.

35 M. Zhang, Y.-P. Chen and H.-C. Zhou, CrystEngComm, 2013, 15, 9544-9552.

36 W. Morris, B. Volosskiy, S. Demir, F. Gándara, P. L. McGrier, H. Furukawa, D. Cascio, J. F. Stoddart and O. M. Yaghi, Inorg. Chem., 2012, 51, 6443-6445.

37 S. Ma, D. Sun, M. Ambrogio, J. A. Fillinger, S. Parkin and H.-C. Zhou, J. Am. Chem. Soc., 2007, 129, 1858-1859.

38 T. A. Makal, A. A. Yakovenko and H.-C. Zhou, J. Phys. Chem. Lett., 2011, 2, 1682-1689.

39 H.-L. Jiang, T. A. Makal and H.-C. Zhou, Coord. Chem. Rev., 2013, 257, 2232-2249.

40 O. K. Farha, C. D. Malliakas, M. G. Kanatzidis and J. T. Hupp, J. Am. Chem. Soc., 2009, 132, 950-952. 
41 M. O'Keeffe and O. M. Yaghi, Chem. Rev., 2011, 112, 675-702.

42 V. A. Blatov, L. Carlucci, G. Ciani and D. M. Proserpio, CrystEngComm, 2004, 6, 378-395.

43 L. Carlucci, G. Ciani and D. M. Proserpio, Coord. Chem. Rev., 2003, 246, 247-289.

44 M. Li, D. Li, M. O'Keeffe and O. M. Yaghi, Chem. Rev., 2013, 114, 1343-1370.

45 H. Chun, D. Kim, D. N. Dybtsev and K. Kim, Angew. Chem., Int. Ed., 2004, 43, 971-974.

46 O. Delgado-Friedrichs, M. O'Keeffe and O. M. Yaghi, Acta Crystallogr., Sect. A: Found. Crystallogr., 2006, 62, 350-355.

47 O. Delgado-Friedrichs, M. O'Keeffe and O. M. Yaghi, Phys. Chem. Chem. Phys., 2007, 9, 1035-1043.

48 O. Delgado Friedrichs, M. O'Keeffe and O. M. Yaghi, Solid State Sci., 2003, 5, 73-78.

49 T. Muller and S. Brase, RSC Adv., 2014, 4, 6886-6907.

50 S. R. Taylor, Geochim. Cosmochim. Acta, 1964, 28, 1273-1285.

51 J. H. Cavka, S. Jakobsen, U. Olsbye, N. Guillou, C. Lamberti, S. Bordiga and K. P. Lillerud, J. Am. Chem. Soc., 2008, 130, 13850-13851.

52 L. Ma, A. Jin, Z. Xie and W. Lin, Angew. Chem., Int. Ed., 2009, 48, 9905-9908.

53 L. Wen, P. Cheng and W. Lin, Chem. Sci., 2012, 3, 2288-2292.

54 L. Wen, P. Cheng and W. Lin, Chem. Commun., 2012, 48, 2846-2848.

55 M. Dincǎ, A. Dailly, Y. Liu, C. M. Brown, D. A. Neumann and J. R. Long, J. Am. Chem. Soc., 2006, 128, 16876-16883.

56 M. Dincǎ, A. Dailly and J. R. Long, Chem. - Eur. J., 2008, 14, 10280-10285.

57 I. Timokhin, J. Baguna Torres, A. J. P. White, P. D. Lickiss, C. Pettinari and R. P. Davies, Dalton Trans., 2013, 42, 13806-13808.

58 J. M. Gotthardt, K. F. White, B. F. Abrahams, C. Ritchie and C. Boskovic, Cryst. Growth Des., 2012, 12, 4425-4430.

59 Y.-S. Xue, L. Zhou, M.-P. Liu, S.-M. Liu, Y. Xu, H.-B. Du and X.-Z. You, CrystEngComm, 2013, 15, 6229-6236.

60 Y. E. Cheon and M. P. Suh, Chem. Commun., 2009, 2296-2298.

61 J. Tian, R. K. Motkuri, P. K. Thallapally and B. P. McGrail, Cryst. Growth Des., 2010, 10, 5327-5333.

62 T.-F. Liu, J. Lü, C. Tian, M. Cao, Z. Lin and R. Cao, Inorg. Chem., 2011, 50, 2264-2271.

63 J. Tian, L. V. Saraf, B. Schwenzer, S. M. Taylor, E. K. Brechin, J. Liu, S. J. Dalgarno and P. K. Thallapally, J. Am. Chem. Soc., 2012, 134, 9581-9584.

64 L.-L. Liang, S.-B. Ren, J. Wang, J. Zhang, Y.-Z. Li, H.-B. Du and X.-Z. You, CrystEngComm, 2010, 12, 2669-2671.

65 Y.-X. Li, M. Xue, L. Huang, S.-R. Chen and S.-L. Qiu, Chem. Res. Chin. Univ., 2013, 29, 611-616.

66 F. Nouar, J. F. Eubank, T. Bousquet, L. Wojtas, M. J. Zaworotko and M. Eddaoudi, J. Am. Chem. Soc., 2008, 130, 1833-1835.

67 G. K. H. Shimizu, Nat. Chem., 2010, 2, 909-911.

68 M. Eddaoudi, J. Kim, J. B. Wachter, H. K. Chae, M. O'Keeffe and O. M. Yaghi, J. Am. Chem. Soc., 2001, 123, 4368-4369.
69 J.-R. Li, A. A. Yakovenko, W. Lu, D. J. Timmons, W. Zhuang, D. Yuan and H.-C. Zhou, J. Am. Chem. Soc., 2010, 132, 17599-17610.

70 J.-R. Li and H.-C. Zhou, Nat. Chem., 2010, 2, 893-898.

71 M. Zhang, W. Lu, J.-R. Li, M. Bosch, Y.-P. Chen, T.-F. Liu, Y. Liu and H.-C. Zhou, Inorg. Chem. Front., 2014, 1, 159-162.

72 D. Zhao, D. Yuan, D. Sun and H.-C. Zhou, J. Am. Chem. Soc., 2009, 131, 9186-9188.

73 D. Yuan, D. Zhao, D. Sun and H.-C. Zhou, Angew. Chem., Int. Ed., 2010, 49, 5357-5361.

74 Y. Yan, X. Lin, S. Yang, A. J. Blake, A. Dailly, N. R. Champness, P. Hubberstey and M. Schroder, Chem. Commun., 2009, 1025-1027.

75 Y. Yan, I. Telepeni, S. Yang, X. Lin, W. Kockelmann, A. Dailly, A. J. Blake, W. Lewis, G. S. Walker, D. R. Allan, S. A. Barnett, N. R. Champness and M. Schröder, J. Am. Chem. Soc., 2010, 132, 4092-4094.

76 Y. Yan, S. Yang, A. J. Blake, W. Lewis, E. Poirier, S. A. Barnett, N. R. Champness and M. Schroder, Chem. Commun., 2011, 47, 9995-9997.

77 O. K. Farha, A. Özgür Yazaydın, I. Eryazici, C. D. Malliakas, B. G. Hauser, M. G. Kanatzidis, S. T. Nguyen, R. Q. Snurr and J. T. Hupp, Nat. Chem., 2010, 2, 944-948.

78 O. K. Farha, I. Eryazici, N. C. Jeong, B. G. Hauser, C. E. Wilmer, A. A. Sarjeant, R. Q. Snurr, S. T. Nguyen, A. Ö. Yazaydın and J. T. Hupp, J. Am. Chem. Soc., 2012, 134, 15016-15021.

79 W. Lu, D. Yuan, T. A. Makal, J.-R. Li and H.-C. Zhou, Angew. Chem., Int. Ed., 2012, 51, 1580-1584.

80 W. Lu, D. Yuan, T. A. Makal, Z. Wei, J.-R. Li and H.-C. Zhou, Dalton Trans., 2013, 42, 1708-1714.

81 J.-R. Li, J. Yu, W. Lu, L.-B. Sun, J. Sculley, P. B. Balbuena and H.-C. Zhou, Nat. Commun., 2013, 4, 1538.

82 Z. J. Zhang, Y. G. Zhao, Q. H. Gong, Z. Li and J. Li, Chem. Commun., 2013, 49, 653-661.

83 M. Zhang, Z. Perry, J. Park and H.-C. Zhou, Polymer, 2014, 55, 335-339.

84 P. D. C. Dietzel, R. Blom and H. Fjellvåg, Eur. J. Inorg. Chem., 2008, 2008, 3624-3632.

85 D. Britt, H. Furukawa, B. Wang, T. G. Glover and O. M. Yaghi, Proc. Natl. Acad. Sci. U. S. A., 2009, 106, 20637-20640.

86 D.-A. Yang, H.-Y. Cho, J. Kim, S.-T. Yang and W.-S. Ahn, Energy Environ. Sci., 2012, 5, 6465-6473.

87 A. R. Millward and O. M. Yaghi, J. Am. Chem. Soc., 2005, 127, 17998-17999.

88 S. R. Caskey, A. G. Wong-Foy and A. J. Matzger, J. Am. Chem. Soc., 2008, 130, 10870-10871.

89 M. Wriedt, J. P. Sculley, A. A. Yakovenko, Y. Ma, G. J. Halder, P. B. Balbuena and H.-C. Zhou, Angew. Chem., Int. Ed., 2012, 51, 9804-9808.

90 H. Wu, J. M. Simmons, Y. Liu, C. M. Brown, X. S. Wang, S. Ma, V. K. Peterson, P. D. Southon, C. J. Kepert, H. C. Zhou, T. Yildirim and W. Zhou, Chem. - Eur. J., 2010, 16, 5205-5214. 
91 H. Wu, Q. Gong, D. H. Olson and J. Li, Chem. Rev., 2012, 112, 836-868.

92 Z. R. Herm, B. M. Wiers, J. A. Mason, J. M. van Baten, M. R. Hudson, P. Zajdel, C. M. Brown, N. Masciocchi, R. Krishna and J. R. Long, Science, 2013, 340, 960-964.

93 J. Park, Z. U. Wang, L.-B. Sun, Y.-P. Chen and H.-C. Zhou, J. Am. Chem. Soc., 2012, 134, 20110-20116.

94 A. Schaate, P. Roy, A. Godt, J. Lippke, F. Waltz, M. Wiebcke and P. Behrens, Chem. - Eur. J., 2011, 17, 6643-6651.

95 X. Lin, I. Telepeni, A. J. Blake, A. Dailly, C. M. Brown, J. M. Simmons, M. Zoppi, G. S. Walker, K. M. Thomas, T. J. Mays, P. Hubberstey, N. R. Champness and M. Schroder, J. Am. Chem. Soc., 2009, 131, 2159-2171.

96 T. Tsuruoka, S. Furukawa, Y. Takashima, K. Yoshida, S. Isoda and S. Kitagawa, Angew. Chem., Int. Ed., 2009, 48, 4739-4743.

97 H. Wu, Y. S. Chua, V. Krungleviciute, M. Tyagi, P. Chen, T. Yildirim and W. Zhou, J. Am. Chem. Soc., 2013, 135, 10525-10532.
98 F. Vermoortele, B. Bueken, G. Le Bars, B. Van de Voorde, M. Vandichel, K. Houthoofd, A. Vimont, M. Daturi, M. Waroquier, V. Van Speybroeck, C. Kirschhock and D. E. De Vos, J. Am. Chem. Soc., 2013, 135, 11465-11468.

99 J. Park, D. Yuan, K. T. Pham, J.-R. Li, A. Yakovenko and H.-C. Zhou, J. Am. Chem. Soc., 2011, 134, 99-102.

100 J. W. Brown, B. L. Henderson, M. D. Kiesz, A. C. Whalley, W. Morris, S. Grunder, H. Deng, H. Furukawa, J. I. Zink, J. F. Stoddart and O. M. Yaghi, Chem. Sci., 2013, 4, 2858-2864.

101 J. Park, J.-R. Li, Y.-P. Chen, J. Yu, A. A. Yakovenko, Z. U. Wang, L.-B. Sun, P. B. Balbuena and H.-C. Zhou, Chem. Commun., 2012, 48, 9995-9997.

102 D. Feng, Z.-Y. Gu, J.-R. Li, H.-L. Jiang, Z. Wei and H.-C. Zhou, Angew. Chem., Int. Ed., 2012, 51, 10307-10310.

103 D. Feng, W.-C. Chung, Z. Wei, Z.-Y. Gu, H.-L. Jiang, Y.-P. Chen, D. J. Darensbourg and H.-C. Zhou, J. Am. Chem. Soc., 2013, 135, 17105-17110.

104 Q. Wang, Z. Yang, X. Zhang, X. Xiao, C. K. Chang and B. Xu, Angew. Chem., Int. Ed., 2007, 46, 4285-4289. 